\title{
MEtop-a generator for single top production via FCNC interactions
}

\author{
Rita Coimbra $^{1, \mathrm{a}}$, António Onofre ${ }^{2, \mathrm{~b}}$, Rui Santos ${ }^{3,4, \mathrm{c}}$, Miguel Won ${ }^{1, \mathrm{~d}}$ \\ ${ }^{1}$ LIP/Departamento de Física, Universidade de Coimbra, 3004-516 Coimbra, Portugal \\ ${ }^{2}$ Departamento de Física, Universidade do Minho, 4710-057 Braga, Portugal \\ ${ }^{3}$ Instituto Superior de Engenharia de Lisboa, 1959-007 Lisbon, Portugal \\ ${ }^{4}$ Centro de Física Teórica e Computacional, Universidade de Lisboa, 1649-003 Lisbon, Portugal
}

Received: 22 August 2012 / Revised: 29 October 2012 / Published online: 15 November 2012

(C) The Author(s) 2012. This article is published with open access at Springerlink.com

\begin{abstract}
We present a generator for single top-quark production via flavour-changing neutral currents. The MEtop event generator allows for Next-to-Leading-Order direct top production $p p \rightarrow t$ and Leading-Order production of several other single top processes. A few packages with definite sets of dimension six operators are available. We discuss how to improve the bounds on the effective operators and how well new physics can be probed with each set of independent dimension six operators.
\end{abstract}

\section{Introduction}

The Large Hadron Collider (LHC) at CERN has resumed operation with its center-of-mass energy increased to $8 \mathrm{TeV}$. The LHC top factory will allow us to scrutinise the heaviest of all known quarks with unprecedented precision. Flavour physics is on the top of the agenda as one of most interesting research topic that can be addressed at this collider, through the study of flavour-changing neutral currents (FCNC) in top-quark production and decay. In fact, a wide variety of models shows a strong dependence in the measurable FCNC quantities: for instance, top-quark FCNC branching ratios can vary from extremely small in the Standard Model (SM) to measurable values at the LHC in a variety of the SM extensions [1]. Therefore, the large number of top quarks being produced provide a means to search for deviation from the SM, however small they are. It is clear that the simplest way to search for new FCNC physics is to look for the rare top decays as for example in $t \rightarrow q \gamma$ where $q=u, c$

\footnotetext{
a e-mail: rita.coimbra@ coimbra.lip.pt

b e-mail: Antonio.Onofre@cern.ch

c e-mail: rsantos@cii.fc.ul.pt

de-mail: miguel.won@ coimbra.lip.pt
}

is the up-quark or a c-quark. Limits on the $B R(t \rightarrow q \gamma)$ were set indirectly at LEP [2-5] and HERA [6, 7] and directly at the Tevatron [8] (see [9] for references and details). Presently, the best bound on the photon FCNC current is the one from HERA while the best experimental bounds on $B R(t \rightarrow q Z)$ were obtained at the Tevatron $[10,11]$ and at the LHC $[12,13]$. Finally, the best bound on the strong FCNC current $t q g$ was recently obtained in direct top production at the LHC [14].

Our goal is to provide a tool to measure FCNC related to the top quark at the production level. More evolved top FCNC searches can only be performed if a dedicated generator for top FCNC studies is available. Events for direct top production $p p(g u+g c) \rightarrow t$ studies can be generated at leading order by the PROTOS generator [15], the TopReX generator [16] and also by multi-purpose generators such as CompHEP [17] (see for instance the analysis in [18]), CalcHEP [19, 20] or MadGraph [21]. We considered that it was both necessary and timely to make available a generator for top FCNC physics that included a larger set of FCNC operators together with a larger set of physical processes at the production level. This is the purpose of the MEtop event generator.

MEtop main process is direct top production, $p p(g u+$ $g c) \rightarrow t$, but calculated at Next-to-Leading-Order (NLO). NLO direct top production was implemented by adopting an effective NLO approximation as described in [22]. Besides direct top, MEtop can be used to generate events at LO for all FCNC processes with a top and a gluon or any quark other than the top in the final state. We plan to include other processes like $p p \rightarrow t V$, with $V=\gamma, Z, W$ in the near future. From the theoretical point of view we will adopt the effective operator formalism as described in [23]. We use a set of dimension six effective operators always involving at least one top quark in the vertex. The set of operators is clas- 
sified in three different groups: strong, electroweak and fourfermion (4F) operators. MEtop comes with several choices of packages where different sets of effective operators are available.

The plan of the paper is as follows. The next section describes the complete set of FCNC operators needed for topphysics. In Sect. 3 we provide a detailed description of the physical processes available in MEtop. Section 4 is devoted to the implementation in MEtop of the effective NLO approximation for direct top production. In Sect. 5 we compare the contributions of the different classes of operators to single top production. Our conclusions are drawn in Sect. 6. There are also three appendixes dealing with more technical issues.

\section{The FCNC operators for top physics}

The effective operator formalism assumes that some general theory which has the SM as its low energy limit can be written as a series in $\Lambda$ with operators obeying the SM symmetries,

$\mathcal{L}=\mathcal{L}^{S M}+\frac{1}{\Lambda} \mathcal{L}^{(5)}+\frac{1}{\Lambda^{2}} \mathcal{L}^{(6)}+O\left(\frac{1}{\Lambda^{3}}\right)$,

where $\mathcal{L}^{S M}$ is the SM Lagrangian and $\mathcal{L}^{(5)}$ and $\mathcal{L}^{(6)}$ contain all the dimension five and six operators respectively. This formalism provides a model-independent parametrisation of physics beyond the SM. This Lagrangian contains only SM fields and therefore any new particles and any new interactions are hidden in the effective operators. The term $\mathcal{L}^{(5)}$ vanishes if baryon and lepton number conservation is imposed.

The complete set of dimension six operators is quite vast. In order to simplify the discussion we classify the operators in three categories: strong FCNC operators [24, 25], the ones generating a vertex of the form $\bar{t} u g$, where $g$ is a gluon and $u$ is an up-quark; electroweak FCNC operators [26, 27], which are the ones giving rise to a vertex with one top-quark, an up-quark and one electroweak gauge boson, and finally $4 \mathrm{~F}$ operators which are Fermi interactions with one top quark and three other quarks.

When writing all allowed dimension six operators obeying the required symmetries of the SM Lagrangian, one readily understands that not all operators are independent [23]. They are related by the equations of motion and also by Fierz transformations. Therefore, the total number of operators can be reduced to a minimum set of independent operators. Moreover, this set can be further reduced when only specific processes are studied, like in our case, where all operators have at least one top quark in the interaction. A minimal set of operators for top-quark physics was discussed in $[24,25,28,29]$ and here we will just present this minimal set according to our classification.
We will start by considering the non- $4 \mathrm{~F}$ operators. As previously stated, we divide these operators in two classes: strong FCNC operators, when the gluonic tensor is involved, and electroweak FCNC operators when electroweak gauge bosons are present in the interaction. We assume that $\mathcal{O}^{i j}$ and $\mathcal{O}^{j i}$ are independent operators and the hermitian conjugate of all the operators are included in the final Lagrangian.

Following the notation of [23], the independent operators contributing to the strong FCNC vertices can be written as

$\mathcal{O}_{u G \phi}^{i j}=\bar{q}_{L}^{i} \lambda^{a} \sigma^{\mu \nu} u_{R}^{j} \tilde{\phi} G^{a \mu \nu}$,

where $G_{\mu \nu}^{a}$ is the gluonic field tensor, $u_{R}^{i}$ stands for a right-handed quark singlet and $q_{L}^{i}$ represents the left-handed quark doublet. FCNC occurs because one of the indices is always equal to 3 while the other is either 1 or 2 , that is, there is always one (and one only) top quark present in the operator; the remaining fermion field in the interaction is either an up or a c-quark. These operators will give rise to the FCNC vertices of the form $g t \bar{u}_{i}$ (with $u_{i}=u, c$ ) and the corresponding hermitian conjugate interaction with an independent coefficient. Operator in (2) also appears in the literature as a dimension 5 operator. In that case, the corresponding FCNC Lagrangian is written as

$$
\begin{aligned}
\mathcal{L}_{\mathcal{S}}= & i \kappa_{u} \frac{g_{s}}{\Lambda} \bar{u} \lambda^{a} \sigma^{\mu \nu}\left(f_{u}+h_{u} \gamma_{5}\right) t G_{\mu \nu}^{a} \\
& +(u \leftrightarrow c)+\text { h.c. }
\end{aligned}
$$

where $\kappa_{u}$ is real, $g_{s}$ is the strong coupling and $f_{u}$ and $h_{u}$ are complex numbers with $\left|f_{u}\right|^{2}+\left|h_{u}\right|^{2}=1$ (see Appendix A for a detailed discussion relating the forms of the strong FCNC operators).

In the electroweak sector we now have to look for FCNC vertices of the type $V t \bar{u}_{i}$ (with $u_{i}=u, c$ and $V=Z, \gamma$ ). The minimal set of operators that give rise to the above interactions can be written as

$$
\begin{aligned}
& \mathcal{O}_{u B \phi}^{i j}=\bar{q}_{L}^{i} \sigma^{\mu v} u_{R}^{j} \tilde{\phi} B_{\mu \nu}, \\
& \mathcal{O}_{u W \phi}^{i j}=\bar{q}_{L}^{i} \tau_{I} \sigma^{\mu v} u_{R}^{j} \tilde{\phi} W_{\mu \nu}^{I}, \\
& \mathcal{O}_{\phi u}^{i j}=i\left(\phi^{\dagger} D_{\mu} \phi\right)\left(\bar{u}_{R}^{i} \gamma^{\mu} u_{R}^{j}\right), \\
& \mathcal{O}_{\phi q}^{(1), i j}=i\left(\phi^{\dagger} D_{\mu} \phi\right)\left(\bar{q}_{L}^{i} \gamma^{\mu} q_{L}^{j}\right), \\
& \mathcal{O}_{\phi q}^{(3), i j}=i\left(\phi^{\dagger} \tau_{I} D_{\mu} \phi\right)\left(\bar{q}_{L}^{i} \gamma^{\mu} \tau_{I} q_{L}^{j}\right), \\
& \mathcal{O}_{u \phi}^{i j}=\left(\phi^{\dagger} \phi\right)\left(\bar{q}_{L}^{i} u_{R}^{j} \tilde{\phi}\right),
\end{aligned}
$$

where $B^{\mu \nu}$ and $W_{\mu \nu}^{I}$ are the $U(1)_{Y}$ and $S U(2)_{L}$ field tensors, respectively. As was shown in [28], for all the operators in (5) and (6), $\mathcal{O}^{i j}$ and $\mathcal{O}^{j i}$ are not independent. In fact, by writing the combinations $\mathcal{O}^{i+j}$ and $\mathcal{O}^{i-j}$ and using the equations of motions, it can be shown that only one of these 
combinations is independent. This means that the number of independent operators in (5) and (6) is reduced to three (for each light flavour). The above discussion leads us to the conclusion that the minimal number of non-4F operators needed to study top FCNC physics is 9 for each light flavour ( $u$ and $c$ ).

The equations of motion used to reduce the number of operators relate the operators from the strong and electroweak sectors with the $4 \mathrm{~F}$ operators. The number of independent 4F operators depends on the process considered. Following [30] we found the minimal number of independent $4 \mathrm{~F}$ operators needed for top plus quark production, $p p \rightarrow t q$, which are shown in Appendix B. The final Lagrangian for the study of single top production via FCNC currents can then be written as

$$
\begin{aligned}
& \mathcal{L}_{q q, q g, g g \rightarrow t \bar{q}} \\
& =\frac{1}{\Lambda^{2}} \sum_{\substack{i, j=1,3 \\
i, j=2,3 \\
i \neq j}}\left(\alpha_{u G \phi}^{i j} \mathcal{O}_{u G \phi}^{i j}+\alpha_{u W \phi}^{i j} \mathcal{O}_{u W \phi}^{i j}+\alpha_{u B \phi}^{i j} \mathcal{O}_{u B \phi}^{i j}\right. \\
& \quad+\alpha_{\phi u}^{i j} \mathcal{O}_{\phi u}^{i j}+\alpha_{\phi q}^{(3, i j)} \mathcal{O}_{\phi q}^{(3, i j)}+\alpha_{\phi q}^{(1, i j)} \mathcal{O}_{\phi q}^{(1, i j)} \\
& \left.\quad+\alpha_{u \phi} \mathcal{O}_{u \phi}^{i j}\right)+\frac{1}{\Lambda^{2}} \mathcal{L}_{4 f u}+\frac{1}{\Lambda^{2}} \mathcal{L}_{4 f c}
\end{aligned}
$$

where $\mathcal{L}_{4 f u}$ and $\mathcal{L}_{4 f c}$ are described in Appendix B. One should note that in order to keep a manageable number of $4 \mathrm{~F}$ operators we only consider initial states with up-quarks in the hadron colliders case. There are reasons for considering a reduced set of $4 \mathrm{~F}$ operators, namely the ones that have in the initial state either $u u$ and $u \bar{u}$. First, and assuming that all $4 \mathrm{~F}$ coupling constants are of the same order, these initial states provide the largest contribution for the cross section. Second, our main goal is to provide a means to distinguish between operators by analysing different distributions and this can only be done if the number of operators is not too large. The addition of further $4 \mathrm{~F}$ operators will be done in the future if found necessary. The minimal number of $4 \mathrm{~F}$ operators in the case of FCNC $p p \rightarrow t \bar{t}$ was recently considered in [31].

Before ending this section we will briefly discuss the bounds on the coupling constants $\alpha_{i}$. In the effective operator approach, the Lagrangian has the SM symmetries. Therefore, physics of the top-quark is related with B physics. In reference [32] this relation was explored in order to constrain the electroweak FCNC operators. ${ }^{1}$ The most constrained operators are obviously the ones built with quark doublets only while the less constrained are the ones built

\footnotetext{
${ }^{1}$ Other analysis based on B physics observables and electroweak precision constraints were also performed in [33-37] leading to similar conclusions.
}

with quark singlets only. Consequently, for the first generation, bounds on operators with doublets only, $\alpha_{\phi q}^{(3, i j)} / \Lambda^{2}$ and $\alpha_{\phi q}^{(1, i j)} / \Lambda^{2}$, are of the order $0.01 \mathrm{TeV}^{-2}$. For operators $\alpha_{u W \phi}^{u t} / \Lambda^{2}$ and $\alpha_{u B \phi}^{u t} / \Lambda^{2}$ the bounds are of the order $0.3 \mathrm{TeV}^{-2}$ while for $\alpha_{u W \phi}^{t u} / \Lambda^{2}$ and $\alpha_{u B \phi}^{t u} / \Lambda^{2}$ we have $1 \mathrm{TeV}^{-2}$. Finally, regarding operators with singlets only, like $\alpha_{u \phi} / \Lambda^{2}$, reference [32] obtained a bound of the order $3 \mathrm{TeV}^{-2}$. The bounds for operators relating the second and third generation are of the same order of magnitude.

As stated in the introduction, there are new direct bounds from the LHC that lowered the limit on $B R(t \rightarrow q Z)$ to $0.34 \%$ [13]. A new indirect bound from HERA [6] is also available $B R(t \rightarrow q \gamma)<0.5 \%$. Also, a combined study on $\mathrm{B}$ physics and Tevatron data on top-quark production cross section places an indirect bound on the sum of the FCNC branching ratios forcing them to be below the percent level [38]. All these new bounds do not imply any dramatic changes on the bounds in the electroweak sector.

Regarding the strong FCNC operators the most recent search is the one from the ATLAS collaboration [14] in direct top production at the LHC. The upper limits obtained at $95 \% \mathrm{CL}$ for the strong couplings are $\kappa_{u} / \Lambda<$ $6.9 \times 10^{-3} \mathrm{TeV}^{-1}$ and $\kappa_{c} / \Lambda<1.6 \times 10^{-2} \mathrm{TeV}^{-1}$ which in turn can be translated into strong branching ratio bounds $B R(t \rightarrow u g)<5.7 \times 10^{-5}$ and $B R(t \rightarrow c g)<2.7 \times 10^{-4}$.

Contrary to the dimension six FCNC operators from the strong and electroweak sector, there are no useful bounds on the $4 \mathrm{~F}$ operators involving two top-quarks and this is even more so if the top is right-handed. Therefore, the LHC can place constraints on these operators.

\section{Physical processes}

MEtop generates events according to the Von Neumann algorithm (see [39] for details). The amplitudes for each process were generated with CalcHEP [19, 20], and the Feynman rules for the effective operators were derived with LanHEP [40, 41]. Integrations are performed using the CUBA library [42], configured to use VEGAS algorithm [43] Generation of events for hadron colliders need the linking with the LHAPDF package [44]. The events are written in the standard format of Les Houches event file [45] (.LHE). Whenever possible our results were checked via a completely different path. First, the Feynman rules were generated by the implementation of the effective operators in UFO [46]. Then, cross sections calculation and event generation was performed using MadGraph 5 [21]. We always found an excellent agreement with MEtop.

The following hard processes are already included in MEtop: direct top production at LO and NLO both for $p p$ and for $p \bar{p}$ colliders, which at the parton level amounts 
to the processes $g q \rightarrow t$ and $g u \rightarrow g t$ where $q=u, c$; $p p(\bar{p}) \rightarrow q t$, with all possible parton contributions in the initial state taken into account and $q$ is now any quark other than the top-quark. The corresponding conjugate processes, with an anti-top in the final state, are also included, and the processes can be generated independently. There are several available packages in MEtop, each containing a different set of operators. The list of operators in each package is presented in Appendix B.

In Fig. 1 we present direct top production together with the top + gluon processes. The two have in common the fact that only the strong FCNC couplings contribute to the process. Only one diagram contributes to direct top channel while several diagrams with gluon exchange contribute to top + gluon. MEtop allows for generation of events at LO and it is the first generator that produces NLO direct top events. As we have already mentioned, currently, FCNC direct top production events can be generated at $\mathrm{LO}$ with the PROTOS generator.

In Fig. 2 the diagrams for top + quark production are shown. Both $q_{1}, q_{2}$ and $q$ run through all quarks other than the top-quark, that is $u, d, c, s, b$ and the respective antiparticles. Again, conjugate processes are also included. In the diagram on the right, only strong FCNC operators are present. On the left diagram all operators can take part, including the 4F ones, contributing to LO single top production at the parton level. A detailed description of each process will be presented in Appendix C.
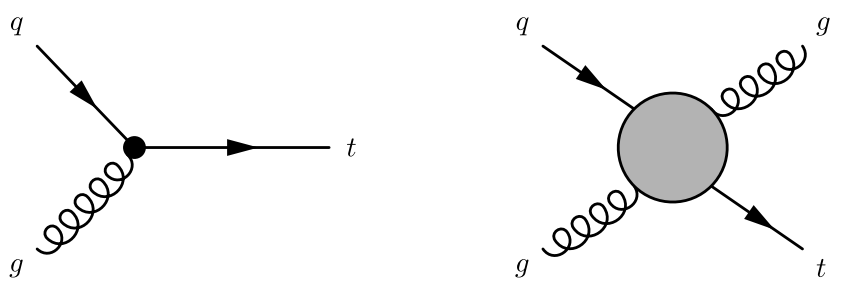

Fig. 1 FCNC leading order direct top production and top + gluon production at the parton level. Only FCNC strong operators contribute to the process
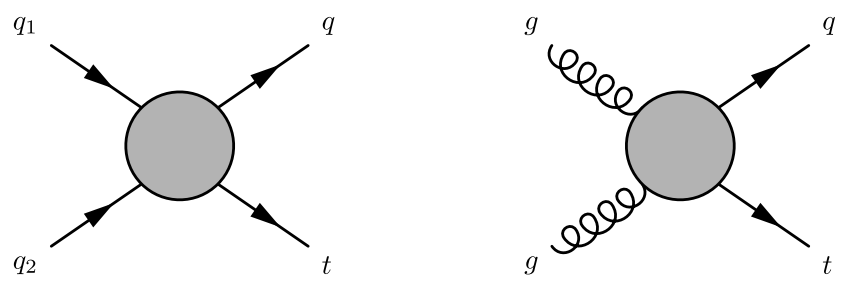

Fig. 2 FCNC leading order top + quark production at the parton level. FCNC strong and electroweak operators contribute to the process together with $4 \mathrm{~F}$ operators

\section{NLO approximation to direct top production}

In an event generator, initial (ISR) and final (FSR) state radiation is included trough a shower mechanism. The shower mechanism assumes collinear factorization, that is, that the real radiation process can be approximated by a branching mechanism, where the first QCD radiation is emitted by one of the legs of the Born configuration. In (9) we present the relation between the transition amplitude for the case of $q \rightarrow q g$ splitting

$$
\begin{aligned}
& \left|M_{n+1}\right|^{2} d \Phi_{n+1} \\
& \quad \Longrightarrow \quad\left|M_{n}\right|^{2} d \Phi_{n} \frac{\alpha_{S}}{2 \pi} \frac{d t}{t} P_{q, q g}(z) d z \frac{d \phi}{2 \pi}
\end{aligned}
$$

where $M_{i}$ is the amplitude and $d \Phi_{i}$ is the phase space for the $i$ th body processes and $P_{q, q g}(z)$ is the Altarelli-Parisi splitting function. This approximation breaks down in the hard $P_{T}$ region where the matrix element $M_{n+1}$ should be used. A factorization prescription or matching scheme is then used to merge these two regions in a smooth and optimised way (see for example the merging approaches CKKW [47] and MLM [48]).

In the previous section we have presented in Fig. 1 the parton level contributions to direct top production, together with the parton level contribution to the hard process $g q \rightarrow$ $g t$. The later process contributes to the inclusive direct top production. The $g q \rightarrow g t$ process has soft and collinear divergences, and this problem can only be solved by including the NLO corrections. Furthermore, the FCNC direct top production cross section was calculated in [49] and a considerable enhancement of about $40 \%$ was found relative to the leading order cross section for the LHC with $\sqrt{s}=14 \mathrm{TeV}$. Therefore it is desirable to have a NLO generator for direct top production at the LHC. It was also shown in [49] that the NLO QCD corrections vastly reduces the dependence of the total cross section in the renormalization and factorization scales which in turn increases the confidence in the predictions.

In order to calculate the inclusive NLO FCNC direct top cross section one has to consider the Born diagram, the virtual contributions and the real emission diagrams. A sketch of the diagrams from each of the above mentioned contribution are shown in Fig. 3. It is well known that at NLO, the integration in the full phase-space gives rise to infrared divergence from the virtual-born interference part and these divergences are cured by the addition of the real emission process. Although easily fixed for a total cross section analytical calculation, this problem is not straightforward to deal with at an event generator level. In fact, there is no way to extract the infrared infinities as it is usually done with dimensional regularization in a theoretical calculation. There are methods to deal with these infinities such as Phase Space Slicing [50-54] and Subtraction Methods [55, 56]. In this 
Fig. 3 Inclusive FCNC direct top production at NLO in QCD

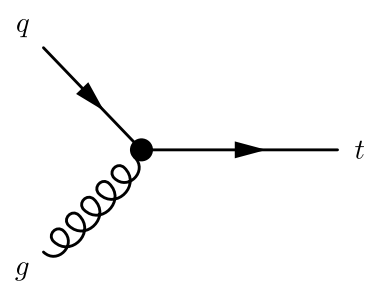

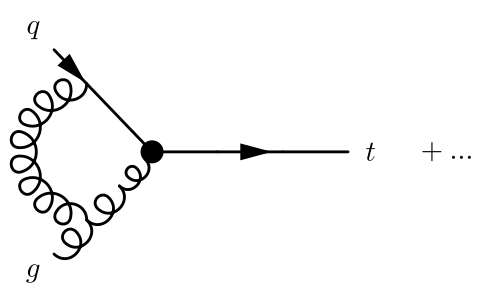

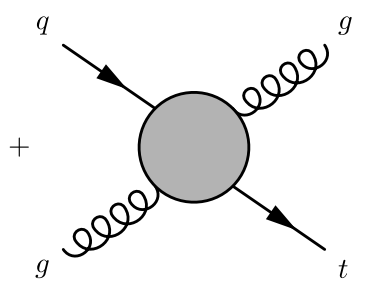

work we will adopt an effective NLO approximation [22] to simulate direct top events at the NLO level. In this approach a merging scheme between $2 \rightarrow 1$ and $2 \rightarrow 2$ events is performed, where each process will separately populate two distinct but joint regions of the phase-space. A resolution parameter must then be defined, which in the present case is the cut in transverse momentum of the top-quark applied to the real radiation process. This $P_{T}^{c u t}$ will then play a role of a matching variable, $P_{T}^{\text {match }}$. The phase-space region for small $P_{T}$ will be described by the $2 \rightarrow 1$ process and the subsequent parton shower (PS) mechanism, whereas the hard $P_{T}$ region will be described by the $2 \rightarrow 2$ process. One must then just make sure that the transition is done in a smooth way. The virtual corrections are included via a Kfactor applied to the cross section of the $2 \rightarrow 1$ process. We assume this to be a good approximation because the kinematics of the Born and Virtual configurations of the direct top process should be very similar. The events will then be generated according to the following relation

$\sigma_{N L O}=K \sigma_{L O}\left(P_{T}^{P S}<P_{T}^{\text {match }}\right)+\sigma_{\text {Real }}\left(P_{T}>P_{T}^{\text {match }}\right)$

where $\sigma_{L O}$ is the tree-level direct top contribution, $\sigma_{\text {Real }}$ is the real radiation part, $K$ is the $K$-factor and $P_{T}^{P S}$ and $P_{T}^{\text {match }}$ are the transverse momentum of the first PS emission and the integration cut of the real radiation process, respectively. Once the direct top events are produced, they will be radiated through a radiator like the one in PYTHIA [57]. In order to avoid double counting, the matching must ensure that the first PS emission from the $2 \rightarrow 1$ process will not fall within the $2 \rightarrow 2$ configuration phase-space. There are two ways of accomplishing it: either by vetoing all radiated $2 \rightarrow 1$ events that would be within the $2 \rightarrow 2$ configuration phase-space or simply by limiting the phase-space region of the radiated $2 \rightarrow 1$ events to the boundaries defined by the resolution variable. We choose to adopt the later.

In order to follow this approach, one must ensure that the PS mechanism added to the generated events from the Born configuration will populate the region with $P_{T}<P_{T}^{\text {match }}$, which can be assured using a $P_{T}$-ordered shower [58], available in both current PYTHIA versions 6.4 and 8.1. We therefore assume that the generated events will be showered by a $P_{T}$-ordered mechanism. Therefore we start by calculating the three cross sections from (10), with $P_{T}^{\text {cut }}=P_{T}^{\text {match }}$ for the $2 \rightarrow 2$ process. For the $\sigma_{N L O}$ cross section we have used the expressions from [49], where the top-quark is onshell. The tree-level direct top and top + gluon amplitudes were generated with CalcHEP where the top-quark and the $\mathrm{W}$ decays were included in order to preserve spin correlations. The cross sections are then calculated with the Cuba library. Hence, the $K$ factor is calculated "on the fly" for each sub-process. After extracting the $K$-factor, the events are generated weighed according to (10). The PS starting scale can then be configured to start the branching in $P_{T}^{\text {match }}$ for the $2 \rightarrow 1$ events configuration, which in MEtop is done by preparing the .LHE files to be used by PYTHIA. A short remark is in order-in the $2 \rightarrow 1$ configuration, no meaningful FSR from the top-quark can be present due to its large mass. Hence, we consider a good approximation to take only ISR into account.

In Fig. 4, the black solid line represents the $P_{T}$ distribution of the top-quark in direct top production, after the first branching in ISR, with starting scale of $m_{t}$. In the same figure, the blue dashed line represents the hard process: top + gluon production. As described previously, $P_{T}$ is the kinematical variable chosen to match the two processes avoiding double counting in the low $P_{T}$ region.

In Fig. 5 we present the $P_{T}$ distribution of the top-quark after the first ISR branching with a $P_{T}^{\text {match }}$ of $10 \mathrm{GeV}$ (left) and $15 \mathrm{GeV}$ (right). The natural criterion to determine the value of the $P_{T}$ matching parameter in the effective NLO approximation is the smoothness of the transverse momentum distribution. There are no significant differences when the value of $P_{T}^{\text {match }}$ is varied in the $5 \mathrm{GeV}$ to $20 \mathrm{GeV}$ range. As can be seen in Fig. 5 , there is never a completely smooth transition between the two sets of events. This effect should 
be included as part of the systematic uncertainties. This feature was checked for a large range of $P_{T}$ match. After including the full shower (ISR + FSR) and Multiple Interaction (MI) we have opted for a value of $P_{T}^{\text {match }}$ of $10 \mathrm{GeV}$.

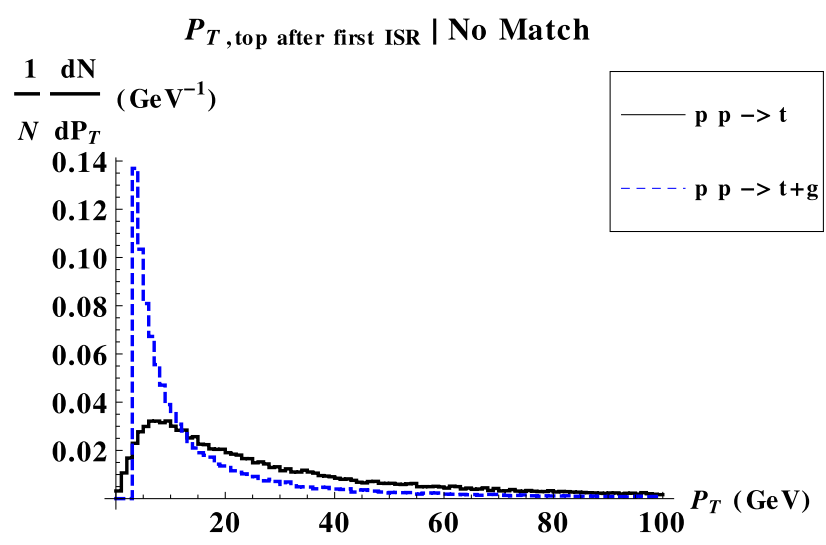

Fig. $4 P_{T}$ distribution of the top-quark for $\sqrt{s}=7 \mathrm{TeV}$. The black solid line is for direct top production after the first branching in ISR, with starting scale of $m_{t}$. The blue dashed line is for the hard process top + gluon production (Color figure online)
In Fig. 6 we show the $P_{T}$ (left) and $\eta$ (right) distributions of the top-quark at the partonic level after the full shower and MI for $P_{T}^{\text {match }}=10 \mathrm{GeV}$. The blue dashed line represents the real radiation part while the grey dashed line is the direct top fully showered but with the $P_{T}$ starting scale at $10 \mathrm{GeV}$. The solid black line is the final NLO distribution which amounts to the sum of the previous two.

In Fig. 7 we present the LO and NLO $P_{T}$ (left) and $\eta$ (right) distributions of the top-quark at the partonic level after the full shower and MI. It is clear from Fig. 7 that the $P_{T}$ and $\eta$ distributions of $\mathrm{LO}$ direct top production are quite different from the corresponding NLO direct top ones. In fact, the distributions show that the use of a constant $K$ factor does not correctly describe the behaviour of direct top at NLO. Hence, a new analysis is needed to improve the accuracy of the bounds on the strong coupling constants $\kappa_{u}$ and $\kappa_{c}$. The direct top NLO $P_{T}$ distribution is shifted to low values of $P_{T}$ as compared to the LO distribution while the $\eta$ distributions are shifted to higher values of $\eta$ as compared to the $\mathrm{LO}$ one. Obviously this applies only to the inclusive direct top analysis. In fact, analysis where a high $P_{T}$ jet is detected alongside the top-quark, like the one performed by
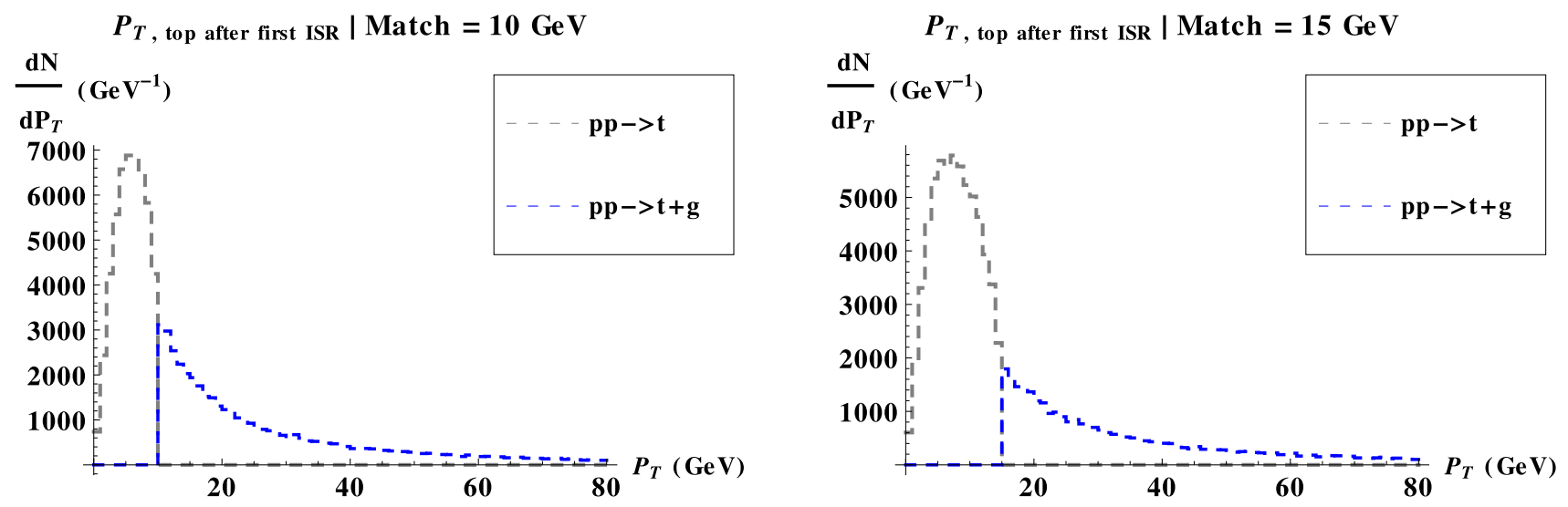

Fig. $5 P_{T}$ distribution of top-quark after the first ISR branching with a $P_{T}^{\text {match }}$ of $10 \mathrm{GeV}($ left $)$ and $15 \mathrm{GeV}$ (right)
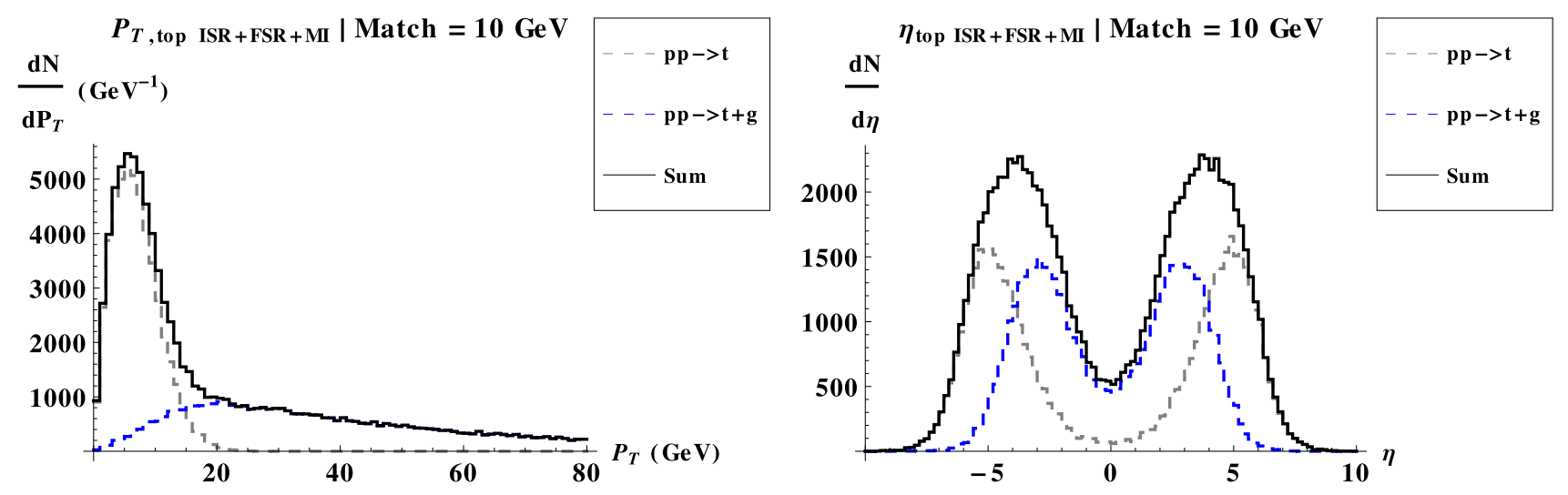

Fig. $6 P_{T}(l e f t)$ and $\eta(r i g h t)$ distributions of the top-quark at the partonic level after the full shower (ISR + FSR) and Multiple Interaction 
the D0 collaboration [18], will not be modified significantly by using MEtop.

The actual experimental analysis is performed by looking at the distributions of the final state particles. Therefore, in Fig. 8 we present the comparison between LO and NLO $P_{T}$ (left) and $\eta$ (right) distributions of the lepton from $t \rightarrow b W \rightarrow b l v$ at the partonic level after the full shower and MI. Again, it is clear that the level of improvement by considering the NLO distributions heavily depends on the particular analysis being performed.

Finally, in Fig. 9 we compare the LO and NLO $P_{T}$ (left) and $\eta$ (right) distributions of the b-quark coming from $t \rightarrow$ $b W \rightarrow b l v$ at the partonic level after the full shower and MI.

We have just described how we generate a sample of inclusive direct top production at NLO. However, if the goal is to set a limit on the strong FCNC coupling, one needs to add the events generated in the process $p p \rightarrow t+$ jet composed by the parton level processes $g g \rightarrow t+q$ and $q_{1} q_{2} \rightarrow t+q$, where $q, q_{1}, q_{2}$ are quarks other than the top and hermitian conjugate reactions are included. Although the main contribution to top + jet production comes from the reac- tion where the jet is a gluon, all processes where the strong FCNC operator intervenes should be taken into account in all analysis. As these processes also suffer from infrared and collinear divergences, we have decided to avoid them by using a similar cut to that of $P_{T}^{\text {match }}$, that is, $P_{T}>10 \mathrm{GeV}$. The complete NLO QCD corrections to the FCNC process of top + jet production were presented in [59]. The corrections can increase the cross section by $10 \%$ to $30 \%$ at the LHC@14TeV.

When generating the top + quark subprocesses we have to decide what is considered as signal in our analysis. $p p \rightarrow$ $t q$ has three different classes of subprocesses: the ones which are exclusive to the Standard Model, like $u \bar{b} \rightarrow t \bar{b}$ (Fig. 10, left), the ones that are originated exclusively via FCNC interactions, e.g. $u u \rightarrow t u$ (Fig. 11), and the ones where interference between SM diagrams and pure FCNC diagrams occurs, like $u \bar{b} \rightarrow t \bar{b}$ (Fig. 10, right).

We define as FCNC signal the contributions from the two later classes of subprocesses. For the pure FCNC processes this poses no problems. However, for the interference terms this procedure leads to the inclusion of a small
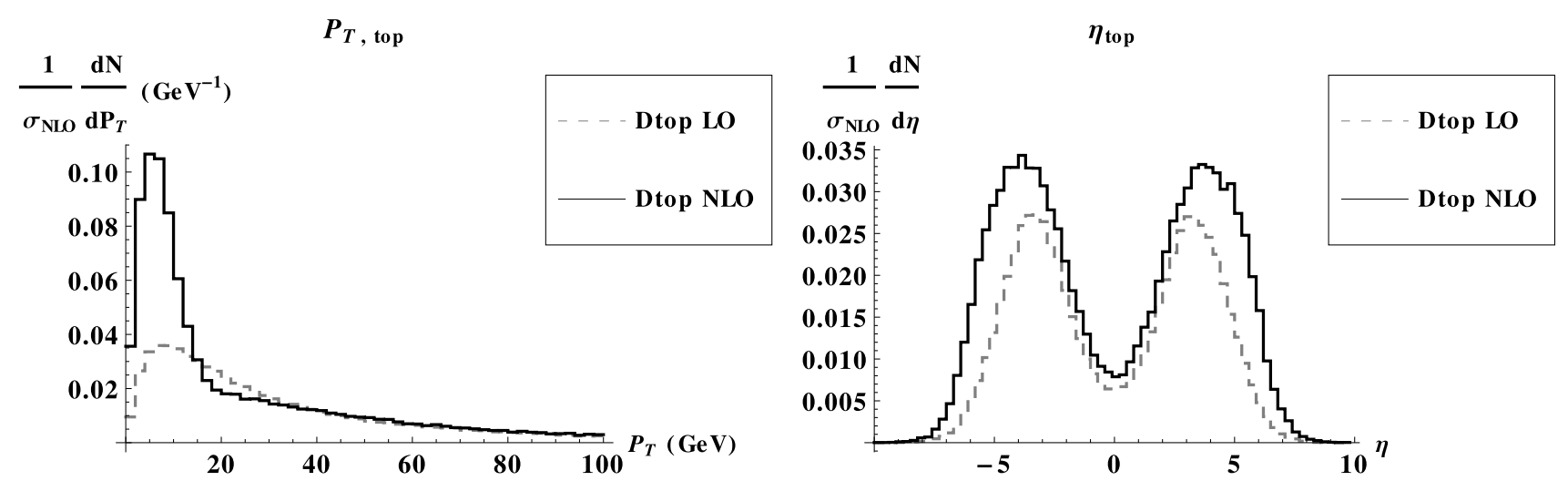

Fig. 7 Comparison of the LO and NLO $P_{T}$ (left) and $\eta$ (right) distributions of the top-quark at the partonic level after the full shower (ISR + FSR) and Multiple Interaction
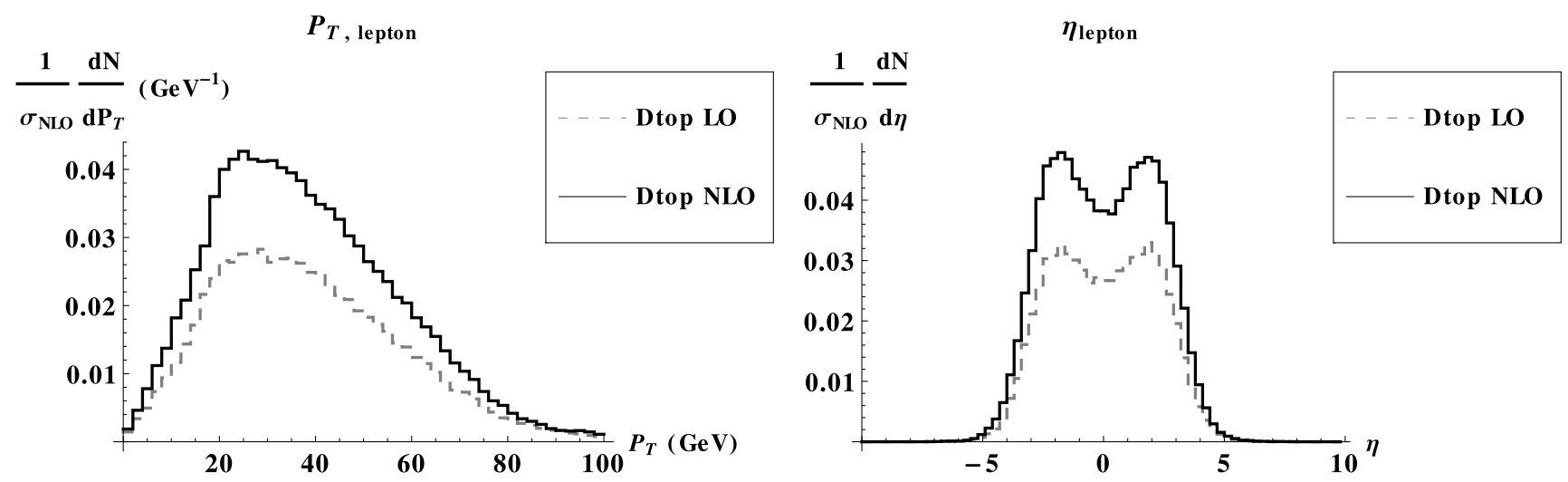

Fig. 8 Comparison of the LO and NLO $P_{T}$ (left) and $\eta(r i g h t)$ distributions of the lepton from $t \rightarrow b W \rightarrow b l v$ at the partonic level after the full shower (ISR + FSR) and Multiple Interaction 

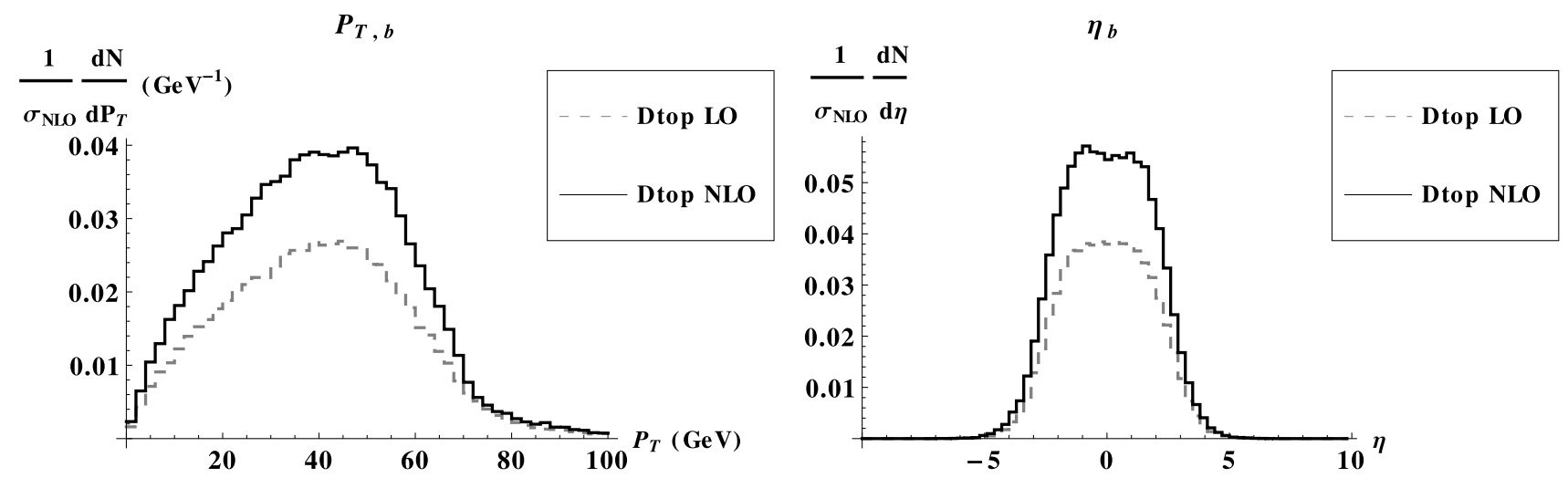

Fig. 9 Comparison of the LO and NLO $P_{T}$ (left) and $\eta$ (right) distributions of the b-quark from $t \rightarrow b W \rightarrow b l v$ at the partonic level after the full shower (ISR + FSR) and Multiple Interaction
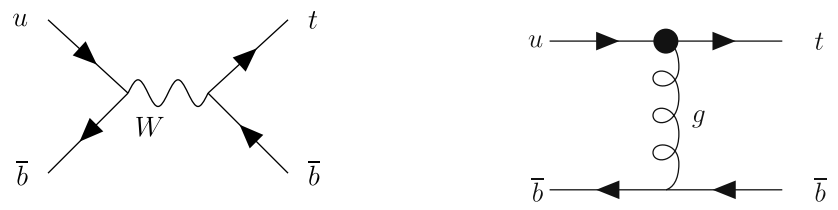

Fig. $10 u \bar{b} \rightarrow t \bar{b}$ contribution to single top production, representing a SM type of diagram (left) and the corresponding strong FCNC diagram (right) that interferes with the SM one

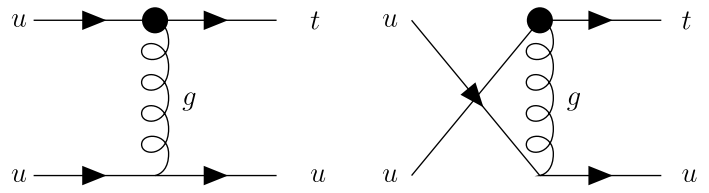

Fig. $11 u u \rightarrow t u$ contribution to single top production as an example of strong FCNC diagrams that do not interfere with the SM ones

portion of events that will also be counted as background. However, choosing the effective strong coupling constants as $\kappa_{u}=0.01(\Lambda=1 \mathrm{TeV}), f_{u}=1 / \sqrt{2}$ and $h_{u}=1 / \sqrt{2}$ and for a $\mathrm{CM}$ energy of $7 \mathrm{TeV}$, the pure $\mathrm{FCNC}$ cross section is $8.718 \mathrm{pb}$, the interference term is $1.205 \mathrm{pb}$ while the SM contribution amounts to only $0.018 \mathrm{pb}$. Hence, the SM contributions can be safely neglected. Note that the diagrams presented in this section are just examples of the three type of diagrams in single top FCNC production-all diagrams with a top quark in the final state are included in MEtop.

In Fig. 12 we show the $P_{T}$ (top) and $\eta$ (bottom) distributions for the direct top at NLO summed with $p p \rightarrow t q$ for a $P_{T}^{\text {match }}=10 \mathrm{GeV}$ and the jet $P_{T}>10 \mathrm{GeV}$. It is clear that the shape of the distributions do not change much with the inclusion of the $p p \rightarrow t q$ process but still the $p p \rightarrow t q$ process gives a contribution of the order of $10 \%$ to the total cross section of the inclusive top production at the LHC at $\sqrt{s}=7 \mathrm{TeV}$.
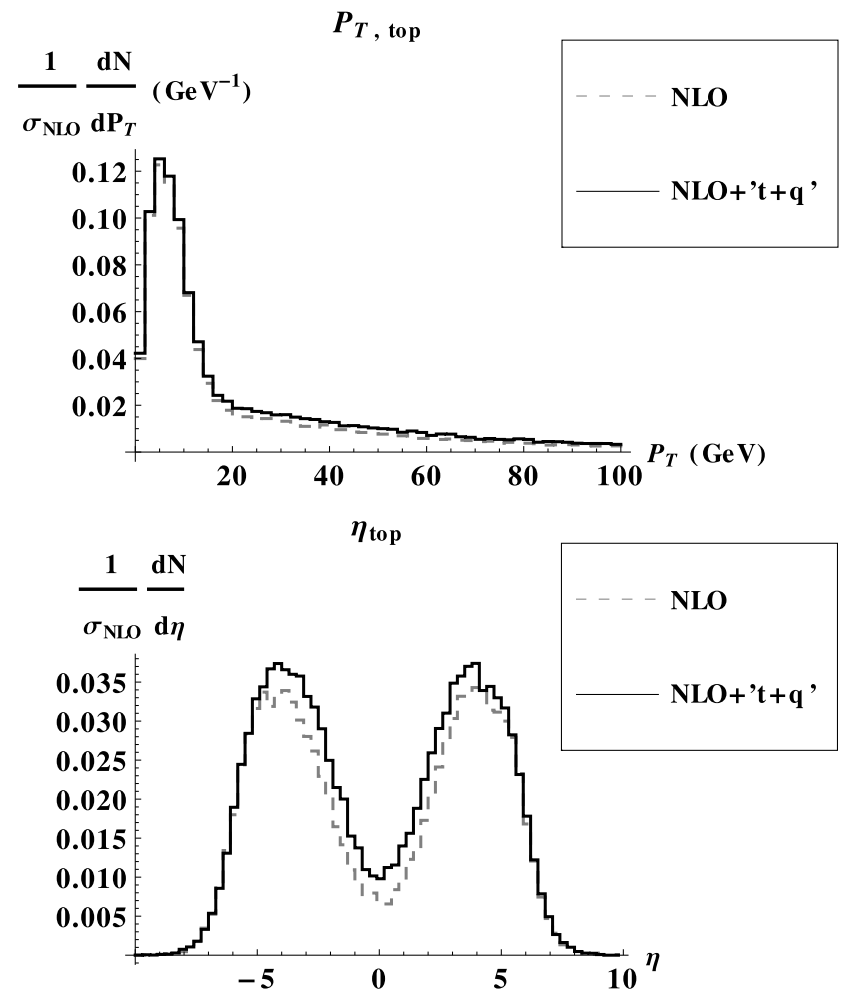

Fig. $12 P_{T}$ (top) and $\eta$ (bottom) distributions of the top-quark for NLO direct top (solid line) and NLO direct top plus $p p \rightarrow t q$ with $P_{T}^{\text {match }}=10 \mathrm{GeV}$ and jet $P_{T}>10 \mathrm{GeV}$

\section{Single top beyond the strong FCNC operators}

In the previous sections we have discussed NLO direct top and $t+q$ production when only the strong FCNC operator is considered. We note that the leading order contribution to direct top does not receive contributions from other operators. Therefore, the NLO calculation is again performed with only the strong FCNC operator. As long as no excess is found at the LHC in the single top channel, the procedure described in the previous section gives us the best possible 
bound on the anomalous strong FCNC coupling when all other operators are discarded.

In the hard $P_{T}$ region, the process $p p \rightarrow t+j e t$ gets contributions from the complete set of independent operators. As these operators are independent from each other (and therefore so are the respective coupling constants) the interference terms between strong and electroweak or $4 \mathrm{~F}$ could be sizeable. If an excess is found in the single top channel, one has to take into account all possible contributions from the remaining operators. A thorough analysis of the distributions of each individual operator will help us understand which operators could be important for a given experimental analysis. Moreover, even if an excess is not seen in the single top channel, dedicated analysis could most probably help constraining definite sets of operators.

We start by considering the strong operator. When all other operators are turned off the $P_{T}$ and $\eta$ distributions have a very mild dependence on the strong coupling constant $\kappa\left(\kappa_{u}\right.$ to be more precise, and we have set $\left.\kappa_{c}=0\right)$. This is shown in Fig. 13 where the $P_{T}$ (left) and $\eta$ (right) distributions of the top-quark are shown for three values of $\kappa$, $0.001,0.01$ and 0.1 and $\Lambda=1 \mathrm{TeV}$. The process is direct top NLO plus $p p \rightarrow t q$ for $\sqrt{s}=7 \mathrm{TeV}$ and $P_{T}^{\text {cut }}=P_{T}^{\text {match }}=$ $10 \mathrm{GeV}$. It is clear that the shape of the distributions does not vary much making it possible to perform the analysis for one constant and then to extract a bound on the strong operator.

We now move to the study of the electroweak operators. We first consider only one operator $O_{u W \phi}$ turned on. At the end of Sect. 2 we showed that present bounds on the coupling constant for this operator are of the order $0.3 \mathrm{TeV}^{-1}$. In Fig. 14 we present the $P_{T}$ (left) and $\eta$ (right) distributions of the top-quark for three values of $\alpha_{u W \phi}, 0.01,0.1$ and 1 and $\Lambda=1 \mathrm{TeV}$. As $\alpha_{u W \phi} \rightarrow 0$ we recover the pure SM contribution of electroweak origin. The SM cross section for this process and for $7 \mathrm{TeV}$ is $\sigma=0.019 \mathrm{pb}$ while the total cross section for $\alpha_{u W \phi}=0.01,0.1$ and 1 are $\sigma=0.0020 \mathrm{pb}$, $0.148 \mathrm{pb}$ and $12.4 \mathrm{pb}$ respectively. Therefore the different shapes of the $P_{T}$ and $\eta$ distributions are due to the interference with the SM contribution. When $\alpha_{u W \phi}=1$, the total cross section is almost 100 times larger than its pure SM counterpart. Therefore, this value shows how the distribution behaves when the SM contribution is negligible.

This kind of behaviour can occur for any operator on the list, provided that the coupling constants are such that SM and FCNC cross sections are of the same order of magnitude. Any deviation relative to the SM showing up in the distributions could mean an interference with one or more operators. Understanding the different distribution requires dedicated studies with no assurance however that the responsible operators could be identified. One should emphasise that a thorough study of the $P_{T}$ and $\eta$ distributions of the top-quark could help identifying classes of FCNC operators. A similar discussion applies to the $4 \mathrm{~F}$ operators case.

One should also note that $p p \rightarrow t q$ does not include direct top, because the strong operator is turned off. Contrary to strong operator scenario, in this case the distributions change with the value of the electroweak constant. Therefore, any bound based on the production process has to take into account that different coupling constants can lead to different distributions.

Having studied the distributions of a definite operator representative of each class, we will now perform a comparison between classes. In Fig. 15 we present the $P_{T}$ (left) and $\eta$ (right) distributions of the top-quark when just one operator is taken non-zero at a time. We compare the distributions of the strong FCNC operator with one electroweak operator (with coupling constant $\alpha_{u W \phi}$ ), and one $4 \mathrm{~F}$ operator, $\left(\bar{u} \gamma_{\mu} \gamma_{L} u\right)\left(\bar{u} \gamma^{\mu} \gamma_{L} t\right)$. It is clear from the figure that the distributions can be quite different and therefore distinguishable

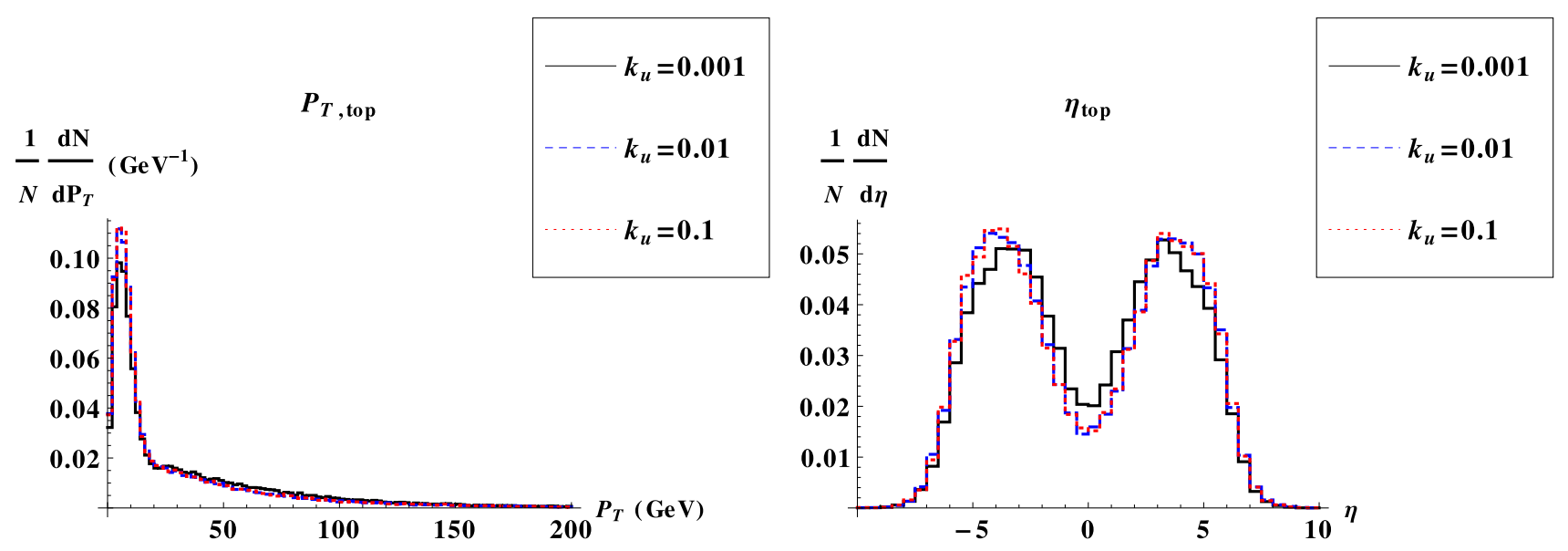

Fig. $13 P_{T}$ (left) and $\eta$ (right) distributions of the top-quark when only the strong operator is turned on with $P_{T}^{\text {cut }}=P_{T}^{\text {match }}=10 \mathrm{GeV}$. Process considered is direct top at NLO plus $p p \rightarrow t q$ for $\sqrt{s}=7 \mathrm{TeV}$ and three values of $\kappa_{u}$ with $\Lambda=1 \mathrm{TeV}$ 


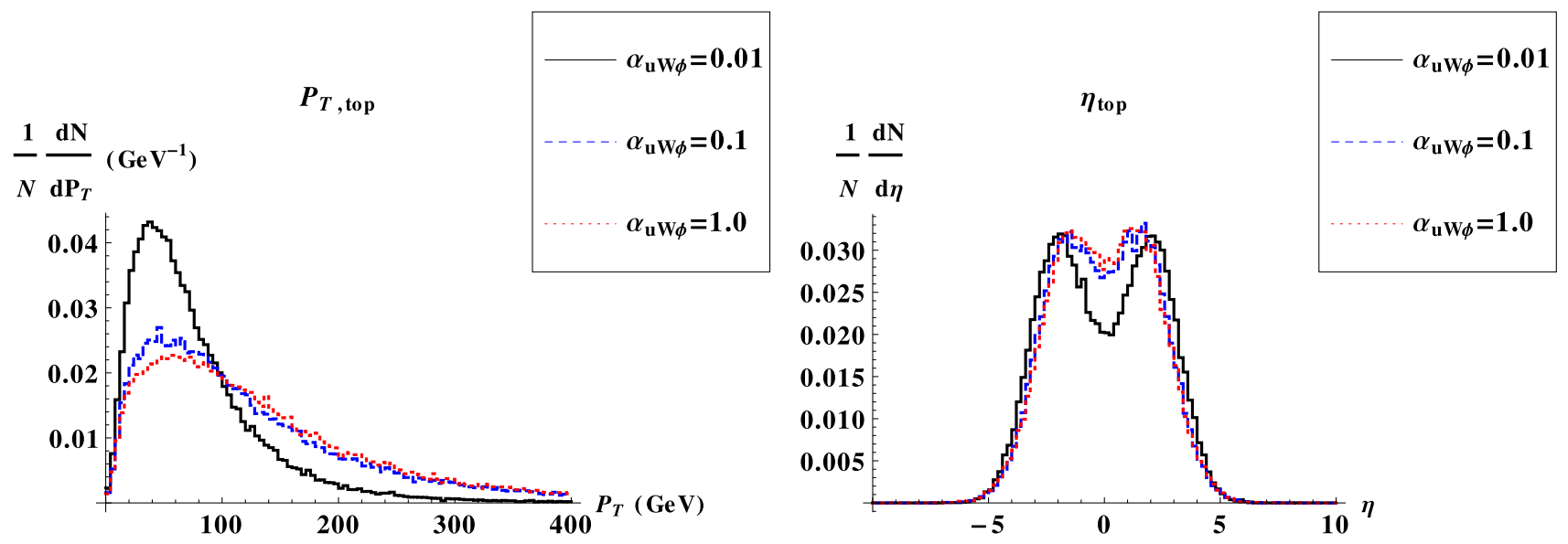

Fig. $14 P_{T}(l e f t)$ and $\eta(r i g h t)$ distributions of the top-quark when just one electroweak operator, $O_{u W \phi}$, is turned on. The process is $p p \rightarrow t q$ for $\sqrt{s}=7 \mathrm{TeV}$ and $P_{T}^{\text {cut }}=10 \mathrm{GeV}$

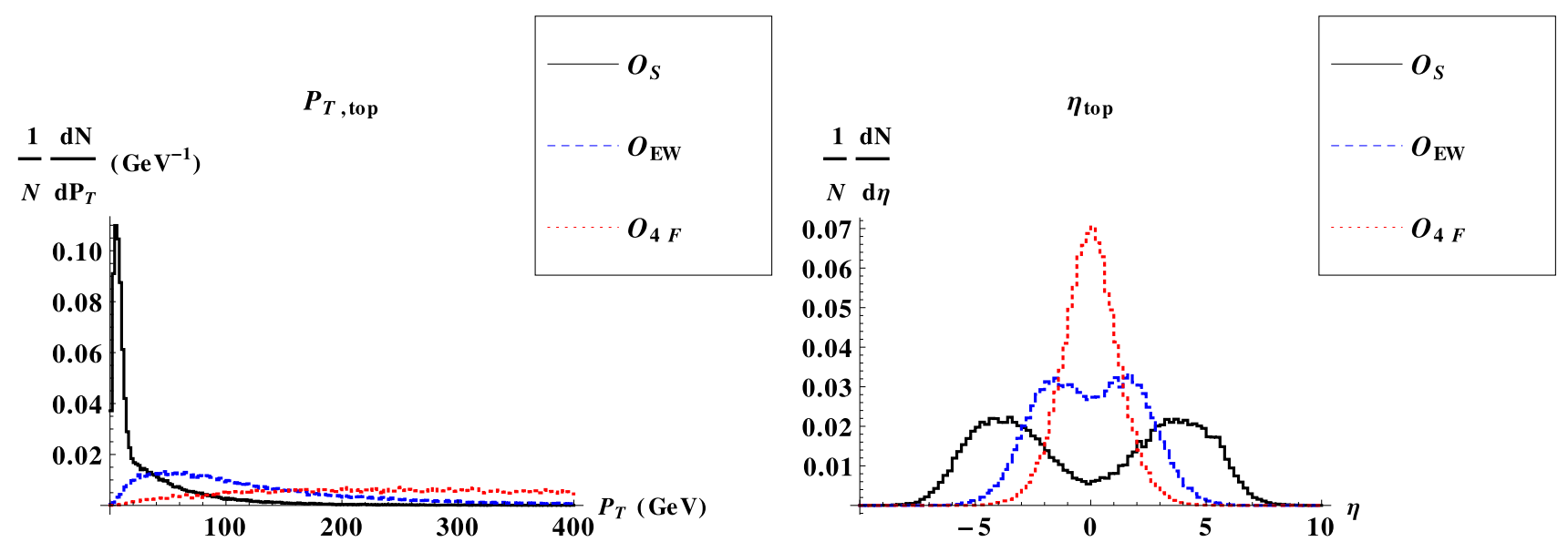

Fig. $15 P_{T}$ (left) and $\eta($ right) distributions of the top-quark when just one operator is taken non-zero at a time. We compare the distributions of the strong FCNC operator with one electroweak, $O_{u W \phi}$, and one $4 \mathrm{~F}$ operator. The process is $p p \rightarrow t q$ for $\sqrt{s}=7 \mathrm{TeV}$ and $P_{T}^{\text {cut }}=10 \mathrm{GeV}$

to some extent. The ability to distinguish the different operators depends heavily on the relative values of the coupling constants. If an excess in single top production is seen we can try to understand its origin by looking at all possible distributions. However, this will always be a hard task because different operators give similar distributions and therefore only very particular scenarios can be probed.

\section{Conclusions}

We have presented a new generator for the study of FCNC top interactions. The generator MEtop comes with different packages, each with a subset of a complete set of dimension six operators. At the moment MEtop can generate events for direct top and for top plus jet production, where the jet can be any quark other than the top or it can be a gluon.
The direct top production process is implemented at NLO using an effective NLO approximation. Also, the inclusive contribution to direct top coming from $p p \rightarrow t q$ can be included in the event generation. We have shown that the top $P_{T}$ and $\eta$ distributions show clear differences when the events are generated at LO or at NLO. Therefore, the use of a constant K-factor does not provide an accurate description of direct top production at NLO. We conclude that a new experimental analysis is needed in order to improve the constraints on the strong FCNC coupling constants. The inclusion of the inclusive process $p p \rightarrow t q$ will further improve this bound. We note that a detailed study of the $P_{T}$ and $\eta$ distributions of the top-quark could help identifying classes of FCNC operators.

At LO, the contributions stemming from the different operators can be compared in the single top production process. In particular, $4 \mathrm{~F}$ operators can be for the first time constrained at hadron colliders. Constraining the $4 \mathrm{~F}$ operators 
can help us understand their role in the asymmetry measured at the Tevatron.

The bounds on $B R(t \rightarrow u(c) Z)$ and $B R(t \rightarrow u(c) \gamma)$ are obtained in the process $p p \rightarrow t \bar{t}$ where one of the top-quarks decays as $t \rightarrow b W$ while the other decays as $t \rightarrow u(c) Z$ or $t \rightarrow u(c) \gamma$. This means that all electroweak FCNC couplings always appear in the same combination. With MEtop we are able to look for distribution that isolates each electroweak FCNC operator. This way more detailed information can be obtained about each operator.

New final states with FCNC contributions, like for instance $p p \rightarrow t W[61]$, are to be included in the next version of MEtop.

Acknowledgements We thank A. Belyaev, A. Semenov, T. Sjöstrand and $\mathrm{Li}$ Lin Yang for discussions. We thank C. Friedrich and D. Hirschbuehl for their help in the debugging and cross-check with existing generators like PROTOS and also for their support.

The work of R.C., A.O., R.S. and M.W. is partially supported by the Portuguese Fundação para a Ciência e a Tecnologia (FCT) under contracts CERN/FP/123619/2011 and PTDC/FIS/117951/2010. R.S. is also partially supported by an FP7 Reintegration Grant, number PERG08-GA-2010-277025 and by PEst-OE/FIS/UI0618/2011. M.W. is also supported by FCT under contract SFRH/BD/45041/2008.

Open Access This article is distributed under the terms of the Creative Commons Attribution License which permits any use, distribution, and reproduction in any medium, provided the original author(s) and the source are credited.

\section{Appendix A: Effective couplings translation}

The representation of the effective coupling constants for each operator is arbitrary. In this appendix we will relate the two most common representations of the strong FCNC coupling constants appearing in the literature. The translation is simple and our goal is to clarify the relation between experimental bounds (and theoretical bounds as well) present in the literature. When the effective strong operator comes from a dimension six effective Lagrangian it is usually represented in the form

$\mathcal{L}_{i j}^{d 6}=\frac{\alpha_{i j}}{\Lambda^{2}} \bar{q}_{L}^{i} \lambda^{a} \sigma^{\mu v} u_{R}^{j} \tilde{\phi} G^{a \mu v}+$ h.c.,

where $\Lambda$ is the scale of new physics. This operator can be written as a dimension five-like operator when the scalar field, $\tilde{\phi}$ is replaced by $(v / \sqrt{2} 0)$, resulting in

$\mathcal{L}_{i j}^{d 6}=\frac{v}{\sqrt{2}} \frac{\alpha_{i j}}{\Lambda^{2}} \bar{u}_{L}^{i} \lambda^{a} \sigma^{\mu v} u_{R}^{j} G^{a \mu v}+$ h.c.

There are in principle four complex constant involved, $\alpha_{i t}$ and $\alpha_{t i}$ with $i=u, c$ in a total of four degrees of freedom. On the other hand, several authors adopt to write the same strong operator as a dimension five effective operator. In this case it is usually written as

$\mathcal{L}_{i j}^{d 5}=-g_{S} \frac{\kappa_{i j}}{\Lambda} \bar{u}^{i} \lambda^{a} \sigma^{\mu \nu}\left(f_{i j}+i h_{i j} \gamma_{5}\right) u^{j} G^{a \mu \nu}+$ h.c.,

where $g_{S}$ is the strong coupling constant, $\kappa_{i j}$ is taken as real and positive, $f_{i j}$ and $h_{i j}$ are complex and $\left|f_{i j}\right|^{2}+\left|h_{i j}\right|^{2}=1$. In both cases $\operatorname{Tr}\left[\lambda_{a} \lambda_{b}\right]=\delta_{a b} / 2$, and the vacuum expectation value is $v=246 \mathrm{GeV}$. Note that in reference [23] (as in other references) the vacuum expectation value is defined as $v=246 / \sqrt{2} \mathrm{GeV}$. The constant $\kappa_{i j}$ is real and only two constants are needed, one for each light flavour; the same is true for the complex constants $f_{i j}$ and $h_{i j}$. Therefore we can use just the light quark index to represent those coupling constants

$\mathcal{L}_{i}^{d 5}=-g_{S} \frac{\kappa_{i}}{\Lambda} \bar{u}^{i} \lambda^{a} \sigma^{\mu \nu}\left(f_{i}+i h_{i} \gamma_{5}\right) u^{j} G^{a \mu \nu}+$ h.c.,

and in this case the index $j$ refers to the top-quark. It is now straightforward to find the relation between the two set of operators

$\alpha_{i t}=-\sqrt{2} g_{S} \frac{\Lambda}{v} \kappa_{i}\left(f_{i}+i h_{i}\right)$

$\alpha_{t i}=-\sqrt{2} g_{S} \frac{\Lambda}{v} \kappa_{i}\left(f_{i}^{*}+i h_{i}^{*}\right)$

with $i=u, c$. In most cases all constants are taken as real. This means that (A.4) can be written as

$\mathcal{L}_{i}^{d 5}=-g_{S} \frac{\kappa_{i}}{\Lambda} \bar{u}^{i} \lambda^{a} \sigma^{\mu \nu}\left(f_{i}+h_{i} \gamma_{5}\right) u^{j} G^{a \mu \nu}+$ h.c.,

and consequently

$\alpha_{i t}=-\sqrt{2} g_{S} \frac{\Lambda}{v} \kappa_{i}\left(f_{i}+h_{i}\right)$

$\alpha_{t i}=-\sqrt{2} g_{S} \frac{\Lambda}{v} \kappa_{i}\left(f_{i}-h_{i}\right)$

with $i=u, c$, all constants are now real and $\left|f_{i}\right|^{2}+\left|h_{i}\right|^{2}=1$.

\section{Appendix B: The complete dimension six Lagrangian for single top production}

As previously discussed, the number of effective dimension six operators is huge. Therefore, no meaningful analysis is possible when all operators are considered simultaneously. Any subset of operators, however small, does not simplify much the task of obtaining information about each individual operator. In order to perform any relevant study involving all different types of $4 \mathrm{~F}$ operators, we have built a $4 \mathrm{~F}$ subset to be used as a basis for our study. Since we are dealing with hadron colliders, our first simplification is to consider only processes initiated by up-quarks. This is equivalent to say that the coupling constants in the $4 \mathrm{~F}$ sector are all 
of the same order. Using references [23, 29, 30] we extract nine $4 \mathrm{~F}$ effective operators that could contribute to single top production at hadron colliders. This set can be written as

$$
\begin{aligned}
\mathcal{O}_{q q}^{i j k l} & =\frac{1}{2}\left(\bar{q}_{L i} \gamma^{\mu} q_{L j}\right)\left(\bar{q}_{L k} \gamma_{\mu} q_{L l}\right), \\
\mathcal{O}_{q q^{\prime}}^{i j k l} & =\frac{1}{2}\left(\bar{q}_{L i a} \gamma^{\mu} q_{L j b}\right)\left(\bar{q}_{L k b} \gamma_{\mu} q_{L l a}\right), \\
\mathcal{O}_{u u}^{i j k l} & =\frac{1}{2}\left(\bar{u}_{R i} \gamma^{\mu} u_{R j}\right)\left(\bar{u}_{R k} \gamma_{\mu} u_{R l}\right), \\
\mathcal{O}_{u d}^{i j k l} & =\left(\bar{u}_{R i} \gamma^{\mu} u_{R j}\right)\left(\bar{d}_{R k} \gamma_{\mu} d_{R l}\right) \\
\mathcal{O}_{u d^{\prime}}^{i j k l} & =\left(\bar{u}_{R i a} \gamma^{\mu} u_{R j b}\right)\left(\bar{d}_{R k b} \gamma_{\mu} d_{R l a}\right), \\
\mathcal{O}_{q u}^{i j k l} & =\left(\bar{q}_{L i} u_{R j}\right)\left(\bar{u}_{R k} q_{L l}\right) \\
\mathcal{O}_{q u^{\prime}}^{i j k l} & =\left(\bar{q}_{L i a} u_{R j b}\right)\left(\bar{u}_{R k b} q_{L l a}\right) \\
\mathcal{O}_{q d}^{i j k l} & =\left(\bar{q}_{L i} d_{R j}\right)\left(\bar{d}_{R k} q_{L l}\right) \\
\mathcal{O}_{q d^{\prime}}^{i j k l} & =\left(\bar{q}_{L i a} d_{R j b}\right)\left(\bar{d}_{R k b} q_{L l a}\right)
\end{aligned}
$$

where $i, j, k, l=1,2,3$ are flavour indices and the subindices $a$ and $b$ indicate the contraction of color indices whenever this pairing is different from the one in spinor contraction. In [30] this set of operators was simplified to the one presented in Table 1 . There is a total of 24 different combinations coming from setting two of the indices $i, j, k$ equal to 1 while the remaining one is set to 1 or 2 . By forcing the initial state to be composed of up-quarks only, we can further reduce the number of operators to 12 (we only allow for one "FCNC-current", $(\bar{u} \Gamma t)$ or $(\bar{c} \Gamma t)$, where $\Gamma$ stands for a generic Lorentz structure). Therefore the final 12 independent 4F operators are the ones obtained by setting $k=j=1$ and $i=1,2$. Because the operators had to be rearranged to allow for the implementation in LanHEP (see discussion below), the final Lagrangian is then written as

$$
\begin{aligned}
& \mathcal{L}_{q q, q g, g g \rightarrow t \bar{q}} \\
& =\frac{1}{\Lambda^{2}} \sum_{\substack{i, j=1,3 \\
\text { or } \\
i, j=2,3 \\
i \neq j}}\left(\alpha_{u G \phi}^{i j} \mathcal{O}_{u G \phi}^{i j}+\alpha_{u W \phi}^{i j} \mathcal{O}_{u W \phi}^{i j}+\alpha_{u B \phi}^{i j} \mathcal{O}_{u B \phi}^{i j}\right. \\
& \quad+\alpha_{\phi u}^{i j} \mathcal{O}_{\phi u}^{i j}+\alpha_{\phi q}^{(3, i j)} \mathcal{O}_{\phi q}^{(3, i j)}+\alpha_{\phi q}^{(1, i j)} \mathcal{O}_{\phi q}^{(1, i j)} \\
& \left.\quad+\alpha_{u \phi} \mathcal{O}_{u \phi}^{i j}\right)+\frac{1}{\Lambda^{2}} \mathcal{L}_{4 f u}+\frac{1}{\Lambda^{2}} \mathcal{L}_{4 f c}
\end{aligned}
$$

where $\mathcal{L}_{4 f u}$ is the $4 \mathrm{~F}$ Lagrangian for anomalous top-up coupling

$$
\begin{aligned}
\mathcal{L}_{4 f u}= & \frac{1}{2}\left(\alpha_{q q}^{1113}+\alpha_{q q^{\prime}}^{1113}\right)\left(\bar{u}_{L} \gamma^{\mu} u_{L}\right)\left(\bar{u}_{L} \gamma_{\mu} t_{L}\right) \\
& -\frac{1}{2}\left(\alpha_{q u^{\prime}}^{1311}+\frac{1}{3} \alpha_{q u}^{1311}\right)\left(\bar{u}_{L} \gamma^{\mu} u_{L}\right)\left(\bar{u}_{R} \gamma_{\mu} t_{R}\right) \\
& -\frac{1}{2}\left(\alpha_{q u^{\prime}}^{1113}+\frac{1}{3} \alpha_{q u}^{1113}\right)\left(\bar{u}_{R} \gamma^{\mu} u_{R}\right)\left(\bar{u}_{L} \gamma_{\mu} t_{L}\right) \\
& +\frac{1}{2} \alpha_{u u}^{1113}\left(\bar{u}_{R} \gamma^{\mu} u_{R}\right)\left(\bar{u}_{R} \gamma_{\mu} t_{R}\right) \\
& -\frac{1}{4} \alpha_{q u}^{1311}\left(\bar{u}_{L} \gamma^{\mu} \lambda^{a} u_{L}\right)\left(\bar{u}_{R} \gamma_{\mu} \lambda^{a} t_{R}\right) \\
& -\frac{1}{4} \alpha_{q u}^{1113}\left(\bar{u}_{R} \gamma^{\mu} \lambda^{a} u_{R}\right)\left(\bar{u}_{L} \gamma_{\mu} \lambda^{a} t_{L}\right)
\end{aligned}
$$

and $\mathcal{L}_{4 f c}$ is the $4 \mathrm{~F}$ Lagrangian for anomalous top-charm coupling

$$
\begin{aligned}
\mathcal{L}_{4 f c}= & \frac{1}{2}\left(\alpha_{q q}^{1123}+\alpha_{q q^{\prime}}^{2113}+\frac{1}{3} \alpha_{q q}^{2113}+\frac{1}{3} \alpha_{q q^{\prime}}^{1123}\right) \\
& \times\left(\bar{u}_{L} \gamma^{\mu} u_{L}\right)\left(\bar{c}_{L} \gamma_{\mu} t_{L}\right) \\
& -\frac{1}{2}\left(\alpha_{q u^{\prime}}^{1321}+\frac{1}{3} \alpha_{q u}^{1321}\right)\left(\bar{u}_{L} \gamma^{\mu} u_{L}\right)\left(\bar{c}_{R} \gamma_{\mu} t_{R}\right) \\
& -\frac{1}{2}\left(\alpha_{q u^{\prime}}^{2113}+\frac{1}{3} \alpha_{q u}^{2113}\right)\left(\bar{u}_{R} \gamma^{\mu} u_{R}\right)\left(\bar{c}_{L} \gamma_{\mu} t_{L}\right) \\
& +\frac{1}{2}\left(\alpha_{u u}^{1123}+\frac{1}{3} \alpha_{u u}^{2113}\right)\left(\bar{u}_{R} \gamma^{\mu} u_{R}\right)\left(\bar{c}_{R} \gamma_{\mu} t_{R}\right) \\
& -\frac{1}{4} \alpha_{q u}^{1321}\left(\bar{u}_{L} \gamma^{\mu} \lambda^{a} u_{L}\right)\left(\bar{c}_{R} \gamma_{\mu} \lambda^{a} t_{R}\right) \\
& -\frac{1}{4} \alpha_{q u}^{2113}\left(\bar{u}_{R} \gamma^{\mu} \lambda^{a} u_{R}\right)\left(\bar{c}_{L} \gamma_{\mu} \lambda^{a} t_{L}\right) \\
& +\frac{1}{4}\left(\alpha_{q q}^{2113}+\alpha_{q q^{\prime}}^{1123}\right)\left(\bar{c}_{L} \gamma^{\mu} \lambda^{a} t_{L}\right)\left(\bar{u}_{L} \gamma^{\mu} \lambda^{a} u_{L}\right) \\
& +\frac{1}{4} \alpha_{u u}^{2113}\left(\bar{c}_{R} \gamma^{\mu} \lambda^{a} t_{R}\right)\left(\bar{u}_{R} \gamma^{\mu} \lambda^{a} u_{R}\right) \\
& +\left(\frac{1}{3} \alpha_{q u^{\prime}}^{2311}+\alpha_{q u}^{2311}\right)\left(\bar{c}_{L} t_{R}\right)\left(\bar{u}_{R} u_{L}\right) \\
& +\frac{1}{2} \alpha_{q u^{\prime}}^{2311}\left(\bar{c}_{L} \lambda^{a} t_{R}\right)\left(\bar{u}_{R} \lambda^{a} u_{L}\right) \\
& \\
&
\end{aligned}
$$

Table 1 4F operators contributing to single top production with parton level processes of the type $u(\bar{u}) u(\bar{u}) \rightarrow t(\bar{t}) u(c \bar{c} \bar{u})$

\begin{tabular}{lc}
\hline$\frac{1}{2}\left(\alpha_{q q}^{k j i 3}+\alpha_{q q^{\prime}}^{i j k 3}\right)\left(\bar{u}_{L k} \gamma^{\mu} u_{L j}\right)\left(\bar{u}_{L i} \gamma_{\mu} t_{L}\right)$ & $-\frac{1}{2} \alpha_{q u^{\prime}}^{k 3 i j}\left(\bar{u}_{L k} \gamma^{\mu} u_{L j}\right)\left(\bar{u}_{R i} \gamma_{\mu} t_{R}\right)$ \\
$-\frac{1}{2} \alpha_{q u^{\prime}}^{i j k 3}\left(\bar{u}_{R k} \gamma^{\mu} u_{R j}\right)\left(\bar{u}_{L i} \gamma_{\mu} t_{L}\right)$ & $\frac{1}{2} \alpha_{u u}^{k j i 3}\left(\bar{u}_{R k} \gamma^{\mu} u_{R j}\right)\left(\bar{u}_{R i} \gamma_{\mu} t_{R}\right)$ \\
$-\frac{1}{2} \alpha_{q u}^{k 3 i j}\left(\bar{u}_{L k a} \gamma^{\mu} u_{L j b}\right)\left(\bar{u}_{R i b} \gamma_{\mu} t_{R a}\right)$ & $-\frac{1}{2} \alpha_{q u}^{i j k 3}\left(\bar{u}_{R k a} \gamma^{\mu} u_{R j b}\right)\left(\bar{u}_{L i a} \gamma_{\mu} t_{L a}\right)$ \\
\hline
\end{tabular}




$$
\begin{aligned}
& +\left(\frac{1}{3} \alpha_{q u^{\prime}}^{1123}+\alpha_{q u}^{1123}\right)\left(\bar{c}_{R} t_{L}\right)\left(\bar{u}_{L} u_{R}\right) \\
& +\frac{1}{2} \alpha_{q u^{\prime}}^{1123}\left(\bar{c}_{R} \lambda^{a} t_{L}\right)\left(\bar{u}_{L} \lambda^{a} u_{R}\right)
\end{aligned}
$$

The operators with the Gell-Mann matrices originate from re-writing the ones where the quark colours indices were explicitly summed. The inclusion of $4 \mathrm{~F}$ operators in MEtop was done by implementing the 4F effective Lagrangian in LanHEP. All 4F operators in Table 1 have four coloured particles converging in one point which is a type of interaction LanHEP is not able to handle automatically due to the complex color flow. Therefore, we had to implement these operators using an auxiliary field mechanism [60], where the 4-color vertex is replaced by 3 -color vertices that when combined in $\mathrm{s}, \mathrm{t}$ and $\mathrm{u}$ channels, will reconstruct the 4-fermion interaction. These 3-color vertices are implemented by introducing the interaction terms in the initial Lagrangian together with a unit mass field with a point-like propagator. An example of how a Lagrangian is written is shown in (B.13) with a vectorial auxiliary field

$$
\begin{aligned}
\mathcal{L}_{4 F}= & \left(\bar{\psi}_{L}^{i} \gamma^{\mu} \psi_{L}^{j}\right)\left(\bar{\psi}_{L}^{k} \gamma_{\mu} \psi_{L}^{l}\right) \rightarrow\left(\bar{\psi}_{L}^{i} \gamma^{\mu} \psi_{L}^{j}\right) X_{\mu} \\
& +X^{\nu}\left(\bar{\psi}_{L}^{k} \gamma_{\nu} \psi_{L}^{l}\right)+\frac{1}{2} X_{\mu} X^{v}
\end{aligned}
$$

where $\psi_{L}$ is a left-handed spinor and $X_{\mu}$ is a spin 1 field that does not propagate.

\section{Appendix C: Using MEtop}

\section{C.1 Installation}

MEtop is written in $\mathrm{C}$ and python and it generates events following the .LHE format. It can therefore be easily interfaced with PYTHIA or Herwig. In order to compile it, you need a $\mathrm{C}$ compiler ${ }^{2}$ and python version 2.6 or later. To run the package you must additionally install

- Cuba Library version 3.0

- LHAPDF version 5.8.6

- Numpy version 1.3.0

The Cuba and LHAPDF library must be available through the library environment variable (for example).

To install MEtop you just have to execute "make" in the main directory.

\footnotetext{
${ }^{2}$ There is one file written in Fortran and therefore you also need a Fortran compiler.
}

\section{C.2 The generator}

\section{C.2.1 param.dat}

In MEtop all parameters are set in one file: "param.dat". Table 2 summarizes the definition of each parameter.

\section{C.2.2 Physical processes}

In addition to the parameters defined in Table 2 there are two more flags in "param.dat" file: "cs" and "Process". The first one dictates whether or not to calculate the cross sections and/or to generate events. The second sets which physical process should be taken into account. If "cs" is set to 0 , the cross sections for all sub-processes defined by the "Process" flag will be calculated and no generation will be performed. The result will be stored in the CS folder, in a csX.txt file, where X can be "Dtop", "Gtop" and "Lqtop". If "cs" is set to 1 , only the event generation will be performed. In this case events are produced according to the calculated cross sections. After generation, the .LHE files will be stored in the Events folder together with a file "runinfo.txt" which stores all information related to the event generation.

Top-quark FCNC interactions were introduced in MEtop through an effective Lagrangian. Depending on which operators are "turned on", different physics will be generated. Two different topologies are available: $2 \rightarrow 1 \rightarrow 3$ and $2 \rightarrow 2 \rightarrow 4 .^{3}$ The first one concerns "Direct top" production, and the second is related to "top + gluon" and "top + light quark". Processes are numbered in Table 3.

Strong FCNC top interactions are included in MEtop through two equivalent effective operators, one for the topup-gluon interaction, and the other for the interaction of the top with a c-quark. In process 1 , only the strong coupling constants are needed. Process 2 has the same effective operators but due to the infrared divergences appearing in top + gluon production a cut in the top-quark transverse momentum has to be set via the variable PTmin. In process 3, top + light quark ${ }^{4}$ production, all operators can contribute, strong, electroweak and 4F. It is now possible to choose which operators to include. Again a value for PTmin has to be chosen.

Process 21 is inclusive direct top production at NLO and again only strong operators intervene. The NLO result is obtained by a matching procedure (as described previously) which depends on one variable, PTmatch, to be chosen by the user. The cross section results are written in three files: "csDtopLO.txt", the LO result for direct top, "csDtopNLO.txt", the NLO increment relative to the LO result $\left(\sigma_{N L O}^{\text {Total }}-\sigma_{L O}^{\text {Total }}\right)$, and "csGtop.txt", the LO cross section for "top + gluon" process with a top quark transverse

\footnotetext{
${ }^{3}$ When "SpCorr" is set to 0 , the top-quark decay will not be performed in MEtop, that is, the generated events will have the topology $2 \rightarrow 1$ and $2 \rightarrow 2$. In this case the spin correlations are lost.

${ }^{4}$ Here light quark stands for the set $u, \bar{u}, d, \bar{d}, c, \bar{c}, s, \bar{s}, b, \bar{b}$.
} 
Table 2 Summary description of "param.dat" file.

\begin{tabular}{ll}
\hline Mx & Particle's masses $(\mathrm{x}=u, d, c, s, b, t, e, \mu, \tau, W, Z, H)$ \\
wx & Particle's Widths $(\mathrm{x}=W, t, Z, H)$ \\
Sx & Values for CKM matrix elements $(\mathrm{x}=12,23,13)$ \\
SW & $\sin \theta_{W}\left(\theta_{W}\right.$ is the Weinberg angle) \\
EE & Electromagnetic coupling constant \\
cox & couplings of the x operator $(\mathrm{x}=1,2, \ldots, 9)$ \\
fx, hx & Chirality parameters from operators co1 and $c o 2$ \\
Q & Factorization scale \\
miuR & Renormalization scale for Direct top at NLO \\
L & Energy scale \\
ECM & Centre of mass Energy \\
PTmatch & $P_{T}$ for matching \\
PTmin & Cut in $P_{T}$ for LO 2 $\rightarrow 2$ processes \\
NEvnts & Number of events to generate \\
pdf & PDF name according to LHAPDF \\
pp & Type of collider: 1 for $p p$ and -1 for $p \bar{p}$ \\
DecMod & Turn on/off W decay modes \\
SpCorr & Turn on/off Spin Correlations \\
ttbar & $t, \bar{t}$ channel. 0- $t$ only; $1-\bar{t}$ only; $2-t$ and $\bar{t}$ \\
seed & Turn random number seed \\
\hline
\end{tabular}

Table 3 Processes available in MEtop

\begin{tabular}{lll}
\hline Process number & Description & Comments \\
\hline 1 & Direct top (LO) & Strong Op. only \\
2 & top + gluon (LO) & Strong Op. only; set PTmin \\
3 & top + quark (LO) & All Op.; set PTmin \\
21 & Direct top (NLO) & Strong Op. only \\
22 & Direct top (NLO) + top + quark (LO) & All Op. \\
\hline
\end{tabular}

momentum above "PTmatch". Therefore the variable "PTmin" is irrelevant for this process. After the generation, the results are stored in one file in the Events folder named "DtopNLO.lhe", containing $2 \rightarrow 1 \rightarrow 3$ and the $2 \rightarrow 2 \rightarrow 4$ configurations. These events constitute the inclusive direct top NLO event generation, and must subsequently be showered by PYTHIA using the $P_{T}$-ordered scheme, in order to complete the matching procedure. Finally, with process 22, MEtop sums process 21 with process 3. The "PTmacth" variable plays the same role as in process 21 and "PTmin" will be the top transverse momentum cut, for the "top + quark" sub-processes.

\section{C.3 Running MEtop}

To run the package you just have to execute the command "./run.py" in the main directory. Care should be taken when changing the values of the physical parameters and/or the process you wish to calculate. In such cases you must always recalculate the value of the cross section. In addition, if you change the process used for the generation, you must be sure that all cross sections pertaining the new process are calculated beforehand. This is mandatory because the generation is done using the $\mathrm{cs}^{*}$.txt files saved in the CS folder.

\section{C.4 Available model files}

At the moment there are three different packages available in MEtop, with a different set of effective operators. The main reason to have the different packages is to make the generation of events faster. The lightest version includes only the strong sector. Then there are two other versions one with strong plus electroweak operators and the other one with strong plus $4 \mathrm{~F}$ operators.

In (C.1) we present the strong FCNC Lagrangian as it is written in the package "MEtop_S_vxx.tar.gz"

$\mathcal{L}_{S}=\operatorname{co} 1 \mathcal{O}_{u G}+\operatorname{co} 2 \mathcal{O}_{c G}+$ h.c. 
Table 4 Coefficient dictionary for $\mathcal{L}_{S E W}$

\begin{tabular}{llll}
\hline $\operatorname{co3} \rightarrow \alpha_{u W}^{u t}$ & $\operatorname{co} 4 \rightarrow \alpha_{u W}^{t u}$ & $\cos \rightarrow \alpha_{u B \phi}^{u t}$ & $\cos \rightarrow \alpha_{u B \phi}^{t u}$ \\
$\operatorname{co} 7 \rightarrow \alpha_{\phi u}^{u t}$ & $\cos \rightarrow \alpha_{\phi u}^{t u}$ & $\operatorname{co} 9 \rightarrow \alpha_{\phi q}^{(3, u t)}$ & $\operatorname{co} 10 \rightarrow \alpha_{\phi q}^{(3, t u)}$ \\
$\operatorname{co} 11 \rightarrow \alpha_{\phi q}^{(1, u t)}$ & $\operatorname{co} 12 \rightarrow \alpha_{\phi q}^{(1, t u)}$ & $\operatorname{co} 13 \rightarrow \alpha_{u \phi}^{u t}$ & $\operatorname{co} 14 \rightarrow \alpha_{u \phi}^{t u}$ \\
\hline
\end{tabular}

Table 5 Coefficient dictionary for $\mathcal{L}_{S 4 F}$

\begin{tabular}{|c|c|c|}
\hline$c o 27 \rightarrow \alpha_{q q}^{1113}+\alpha_{q q^{\prime}}^{1113}$ & $\operatorname{co33} \rightarrow \alpha_{q q}^{1123}+\alpha_{q q^{\prime}}^{2113}$ & $\operatorname{co39} \rightarrow \alpha_{u u}^{1123}$ \\
\hline $\operatorname{co} 28 \rightarrow \alpha_{q u^{\prime}}^{1311}$ & $\operatorname{co3} 4 \rightarrow \alpha_{q q}^{2113}+\alpha_{q q^{\prime}}^{1123}$ & $\operatorname{co} 40 \rightarrow \alpha_{u u}^{2113}$ \\
\hline$c o 29 \rightarrow \alpha_{q u^{\prime}}^{1113}$ & $\operatorname{co3} 5 \rightarrow \alpha_{q u^{\prime}}^{1321}$ & $\operatorname{co} 41 \rightarrow \alpha_{q u^{\prime}}^{2311}$ \\
\hline $\operatorname{co3} 0 \rightarrow \alpha_{u u}^{1113}$ & $\operatorname{co3} 6 \rightarrow \alpha_{q u}^{1321}$ & $\operatorname{co4} 4 \rightarrow \alpha_{q u^{\prime}}^{1123}$ \\
\hline $\operatorname{co3} 1 \rightarrow \alpha_{q u}^{1311}$ & $\operatorname{co3} 3 \rightarrow \alpha_{q u^{\prime}}^{2113}$ & $\operatorname{co} 43 \rightarrow \alpha_{q u}^{2311}$ \\
\hline $\operatorname{co3} 2 \rightarrow \alpha_{q u}^{1113}$ & $\cos 8 \rightarrow \alpha_{q u}^{2113}$ & $\operatorname{co} 44 \rightarrow \alpha_{q u}^{1123}$ \\
\hline
\end{tabular}

with

$$
\begin{aligned}
& \mathcal{O}_{u G}=i \frac{g_{s}}{\Lambda} \bar{u} \lambda^{a} \sigma^{\mu \nu}\left(f u+h u \gamma_{5}\right) t G_{\mu \nu}^{a} \\
& \mathcal{O}_{c G}=i \frac{g_{s}}{\Lambda} \bar{c} \lambda^{a} \sigma^{\mu \nu}\left(f c+h c \gamma_{5}\right) t G_{\mu \nu}^{a}
\end{aligned}
$$

and $c o 1, c o 2, f u, h u, f c, h c$ are real constants to be chosen in the file param.dat. The constants $f_{i}, h_{i}$ allow the choice of different chiralities while $\mathrm{co}_{i}$ are overall normalization constants. Although it usually considered that $f_{i}^{2}+h_{i}^{2}=1$, this relation has to be implemented by the user by a judicious choice of parameters $f_{i}$ and $h_{i}$.

The package "MEtop_SEW_vxx.tar.gz" contains the strong and electroweak sectors. The Lagrangian introduced in this package is

$$
\begin{aligned}
\mathcal{L}_{S E W}= & \mathcal{L}_{S}+\frac{1}{\Lambda^{2}} \sum_{\substack{i, j=1,3 \\
i \neq j}}\left(\alpha_{u W}^{i j} \mathcal{O}_{u W}^{i j}+\alpha_{u B \phi}^{i j} \mathcal{O}_{u B \phi}^{i j}\right. \\
& +\alpha_{\phi u}^{i j} \mathcal{O}_{\phi u}^{i j}+\alpha_{\phi q}^{(3, i j)} \mathcal{O}_{\phi q}^{(3, i j)}+\alpha_{\phi q}^{(1, i j)} \mathcal{O}_{\phi q}^{(1, i j)} \\
& \left.+\alpha_{u \phi} \mathcal{O}_{u \phi}^{i j}\right)
\end{aligned}
$$

where the electroweak operators are

$$
\begin{aligned}
& \mathcal{O}_{u \phi}^{i j}=\left(\phi^{\dagger} \phi\right)\left(\bar{q}_{L i} u_{R j} \tilde{\phi}\right), \\
& \mathcal{O}_{\phi q}^{(1, i j)}=i\left(\phi^{\dagger} D_{\mu} \phi\right)\left(\bar{q}_{L i} \gamma^{\mu} q_{L j}\right) \\
& \mathcal{O}_{\phi q}^{(3, i j)}=i\left(\phi^{\dagger} D_{\mu} \tau^{I} \phi\right)\left(\bar{q}_{L i} \gamma^{\mu} \tau^{I} q_{L j}\right), \\
& \mathcal{O}_{\phi u}^{i j}=i\left(\phi^{\dagger} D_{\mu} \phi\right)\left(\bar{u}_{R i} \gamma^{\mu} u_{R j}\right) \\
& \mathcal{O}_{u W}^{i j}=\left(\bar{q}_{L i} \sigma_{\mu \nu} \tau_{I} u_{R j}\right) \tilde{\phi} W_{\mu \nu}^{I}, \\
& \mathcal{O}_{u B \phi}^{i j}=\left(\bar{q}_{L i} \sigma_{\mu \nu} u_{R j}\right) \tilde{\phi} B_{\mu \nu}
\end{aligned}
$$

and all coupling constants are real. In param.dat all coupling constant have the form $\mathrm{co}_{i}$. The relation between the coupling constants presented in (C.3) and the $\mathrm{co}_{i}$ parameters to be chosen in param.dat is presented in Table 4.

Finally, the file "MEtop_S4F_vxx.tar.gz" contains the strong and $4 \mathrm{~F}$ sector

$\mathcal{L}_{S 4 F}=\mathcal{L}_{S}+\mathcal{L}_{4 f u}+\mathcal{L}_{4 f c}$

where the $4 \mathrm{~F}$ lagrangians were presented in (B.11) and (B.12). The relation between the parameters in (B.11) and (B.12) and the corresponding $\mathrm{Co}_{i}$ parameters in the param.dat file is shown in Table 5.

Finally we note that any combination of parameters can be made in a new package and can be made available upon request. Generator and the different packages can be downloaded at http://coimbra.lip.pt/ miguelwon/MEtop/.

\section{References}

1. J.A. Aguilar-Saavedra, Acta Phys. Pol. B 35, 2695 (2004). arXiv:hep-ph/0409342

2. A. Heister et al. (ALEPH Coll), Phys. Lett. B 543, 173 (2002)

3. J. Abdallah et al. (DELPHI Coll.), Phys. Lett. B 590, 21 (2004)

4. G. Abbiendi et al. (OPAL Coll.), Phys. Lett. B 521, 181 (2001)

5. P. Achard et al. (L3 Coll.), Phys. Lett. B 549, 290 (2002)

6. H. Abramowicz et al. (ZEUS Collaboration), Phys. Lett. B 708, 27 (2012). arXiv:1111.3901 [hep-ex]

7. F.D. Aaron et al. (H1 Collaboration), Phys. Lett. B 678, 450 (2009). arXiv:0904.3876 [hep-ex]

8. F. Abe et al. (CDF Coll.), Phys. Rev. Lett. 80, 2525 (1998)

9. R. Guedes, R. Santos, M. Won, arXiv:1007.2992 [hep-ph]

10. V.M. Abazov et al. (D0 Collaboration), Phys. Lett. B 701, 313 (2011). arXiv:1103.4574 [hep-ex]

11. T. Aaltonen et al. (CDF Collaboration), Phys. Rev. Lett. 101, 192002 (2008). arXiv:0805.2109 [hep-ex]

12. G. Aad et al. (ATLAS Collaboration), arXiv:1206.0257 [hep-ex]

13. CMS Collaboration, CMS-PAS-TOP-11-028, 2011 
14. G. Aad et al. (ATLAS Collaboration), Phys. Lett. B 712, 351 (2012). arXiv:1203.0529 [hep-ex]

15. J.A. Aguilar-Saavedra, Nucl. Phys. B 837, 122 (2010). arXiv: 1003.3173 [hep-ph]

16. S.R. Slabospitsky, L. Sonnenschein, Comput. Phys. Commun. 148, 87 (2002). arxiv:hep-ph/0201292

17. E. Boos et al. (CompHEP Collaboration), Nucl. Instrum. Methods Phys. Res., Sect. A 534, 250 (2004). arxiv:hep-ph/0403113

18. V.M. Abazov et al. (D0 Collaboration), Phys. Lett. B 693, 81 (2010). arXiv:1006.3575 [hep-ex]

19. A. Pukhov et al., arXiv:hep-ph/9908288

20. A. Pukhov, arXiv:hep-ph/0412191

21. J. Alwall, M. Herquet, F. Maltoni, O. Mattelaer, T. Stelzer, J. High Energy Phys. 1106, 128 (2011). arXiv:1106.0522 [hep-ph]

22. E.E. Boos, V.E. Bunichev, L.V. Dudko, V.I. Savrin, A.V. Sherstnev, Phys. At. Nucl. 69, 1317 (2006). Yad. Fiz. 69, 1352 (2006)

23. W. Buchmüller, D. Wyler, Nucl. Phys. B 268, 621 (1986)

24. P.M. Ferreira, O. Oliveira, R. Santos, Phys. Rev. D 73, 034011 (2006). arXiv:hep-ph/0510087

25. P.M. Ferreira, R. Santos, Phys. Rev. D 73, 054025 (2006). arXiv:hep-ph/0601078

26. P.M. Ferreira, R.B. Guedes, R. Santos, Phys. Rev. D 77, 114008 (2008). arXiv:0802.2075 [hep-ph]

27. R.A. Coimbra, P.M. Ferreira, R.B. Guedes, O. Oliveira, A. Onofre, R. Santos, M. Won, Phys. Rev. D 79, 014006 (2009). arXiv:0811.1743 [hep-ph]

28. J.A. Aguilar-Saavedra, Nucl. Phys. B 812, 181-204 (2009). arXiv:0811.3842 [hep-ph]

29. B. Grzadkowski, M. Iskrzynski, M. Misiak, J. Rosiek, J. High Energy Phys. 1010, 085 (2010). arXiv:1008.4884 [hep-ph]

30. J.A. Aguilar-Saavedra, Nucl. Phys. B 843, 638 (2011). [Erratumibid. B 851, 443 (2011)]. arXiv:1008.3562 [hep-ph]

31. S.S. Biswal, S. Mitra, R. Santos, P. Sharma, R.K. Singh, M. Won, arXiv:1201.3668 [hep-ph]

32. P.J. Fox, Z. Ligeti, M. Papucci, G. Perez, M.D. Schwartz, Phys. Rev. D 78, 054008 (2008). arXiv:0704.1482 [hep-ph]

33. J. Drobnak, S. Fajfer, J.F. Kamenik, Phys. Lett. B 701, 234 (2011). arXiv:1102.4347 [hep-ph]

34. B. Grzadkowski, M. Misiak, Phys. Rev. D 78, 077501 (2008). [Erratum-ibid. D 84, 059903 (2011)]. arXiv:0802.1413 [hep-ph]

35. Q.-H. Cao, J. Wudka, C.-P. Yuan, Phys. Lett. B 658, 50 (2007). arXiv:0704.2809 [hep-ph]

36. C. Zhang, N. Greiner, S. Willenbrock, arXiv:1201.6670 [hep-ph]

37. N. Greiner, S. Willenbrock, C. Zhang, Phys. Lett. B 704, 218 (2011). arXiv:1104.3122 [hep-ph]
38. P.M. Ferreira, R. Santos, Phys. Rev. D 80, 114006 (2009). arXiv:0903.4470 [hep-ph]

39. K. Nakamura et al. (Particle Data Group), J. Phys. G 37, 075021 (2010) and 2011 partial update for the 2012 edition

40. A. Semenov, Comput. Phys. Commun. 115, 124 (1998)

41. A. Semenov, Comput. Phys. Commun. 180, 431 (2009). arXiv:0805.0555 [hep-ph]

42. T. Hahn, Comput. Phys. Commun. 168, 78 (2005). arXiv: hep-ph/0404043

43. G.P. Lepage, J. Comput. Phys. 27, 192 (1978)

44. M.R. Whalley, D. Bourilkov, R.C. Group, arXiv:hep-ph/0508110

45. J. Alwall et al., Comput. Phys. Commun. 176, 300 (2007). arXiv:hep-ph/0609017

46. C. Degrande, C. Duhr, B. Fuks, D. Grellscheid, O. Mattelaer, T. Reiter, Comput. Phys. Commun. 183, 1201 (2012). arXiv:1108.2040 [hep-ph]

47. S. Catani, F. Krauss, R. Kuhn, B.R. Webber, J. High Energy Phys. 0111, 063 (2001). arXiv:hep-ph/0109231

48. M.L. Mangano, presentation at the FNAL Matrix Element/Monte Carlo Tuning Working Group, 15 November 2002, http://wwwcpd.fnal.gov/personal/mrenna/tuning/nov2002/mlm.pdf

49. J.J. Liu, C.S. Li, L.L. Yang, L.G. Jin, Phys. Rev. D 72, 074018 (2005). arXiv:hep-ph/0508016

50. H. Baer, J. Ohnemus, J.F. Owens, Phys. Rev. D 40, 2844 (1989)

51. K. Fabricius, G. Kramer, G. Schierholz, I. Schmitt, Z. Phys. C 11, 315 (1981)

52. G. Kramer, B. Lampe, Fortschr. Phys. 37, 161 (1989)

53. W.T. Giele, E.W.N. Glover, Phys. Rev. D 46, 1980 (1992)

54. W.T. Giele, E.W.N. Glover, D.A. Kosower, Nucl. Phys. B 403, 633 (1993)

55. S. Catani, M.H. Seymour, Nucl. Phys. B 485, 291 (1997)

56. S. Catani, S. Dittmaier, M.H. Seymour, Z. Trocsanyi, Nucl. Phys. B 627, 189 (2002)

57. T. Sjostrand, S. Mrenna, P. Skands, J. High Energy Phys. 05, 026 (2006)

58. T. Sjostrand, P.Z. Skands, Eur. Phys. J. C 39, 129 (2005). arXiv:hep-ph/0408302

59. J. Gao, C.S. Li, J.J. Zhang, H.X. Zhu, Phys. Rev. D 80, 114017 (2009). arXiv:0910.4349 [hep-ph]

60. A. Belyaev, C. Leroy, R. Mehdiyev, A. Pukhov, J. High Energy Phys. 0509, 005 (2005). arXiv:hep-ph/0502067

61. S.M. Etesami, M. Mohammadi Najafabadi, Phys. Rev. D 81, 117502 (2010). arXiv:1006.1717 [hep-ph] 